\title{
Networks of information token recurrences derived from genomic sequences may reveal hidden patterns in epidemic outbreaks: A case study of the 2019-nCoV coronavirus.
}

\author{
Markus Luczak-Roesch ${ }^{1 *}$ \\ 1 Victoria University of Wellington, School of Information Management \\ * markus.luczak-roesch@vuw.ac.nz
}

\begin{abstract}
Profiling the genetic evolution and dynamic spreading of viruses is a crucial task when responding to epidemic outbreaks. We aim to devise novel ways to model, visualise and analyse the temporal dynamics of epidemic outbreaks in order to help researchers and other people involved in crisis response to make well-informed and targeted decisions about from which geographical locations and time periods more genetic samples may be required to fully understand the outbreak. Our approach relies on the application of Transcendental Information Cascades to a set of temporally ordered nucleotide sequences, and we apply it to real-world data that was collected during the currently ongoing outbreak of the novel 2019-nCoV coronavirus. We assess information-theoretic and network-theoretic measures that characterise the resulting complex network and identify touching points and temporal pathways that are candidates for deeper investigation by geneticists and epidemiologists.
\end{abstract}

Keywords: epidemic outbreaks, genome analysis, bioinformatics, complex systems, network analysis, sequential data mining

\section{Introduction}

How far a virus will eventually spread at local, regional, national and international levels is of central concern during an epidemic outbreak due to the severe consequences large-scale epidemics have on human well-being 5], the stability and coherence of social systems [11], and the global economy [23. A thorough understanding of the genetic characteristics of a virus is crucial to understand the way it is (or may be) transmitted (e.g. human-to-human transmissibility) in order to be able to anticipate the potential reach of an outbreak and develop effective countermeasures. The recent outbreak of a new type of coronavirus - 2019-nCoV - provides plenty of examples of the way researchers seek to quickly approach the aforementioned problems of genetic decomposition of the virus [2 8 and modelling of its transmission dynamics [15].

Here we present a novel approach to gain insights into the transmission dynamics of an epidemic outbreak. Our method is based on Transcendental Information Cascades 16 17 and combines gene sequence analysis with temporal data mining to uncover potential relationships between a virus' genetic evolution and distinct occurrences of infections. We apply this new approach to publicly available genomic sequence data from the currently ongoing outbreak of the 2019-nCoV coronavirus. 
Figure 1. Example of the unique codon tokenisation used in this study. The example sequence comprises three window steps and shows how the +1 (marked in blue), +2 (marked in orange) and +3 (marked in black) reading frames lead to seven unique codon tokens extracted from the nucleotide sequence.

Our results show pathways of similarity between certain clusters of 2019-nCoV virus samples taken in different geographical locations, and indicate coronavirus cases that are candidates for further investigation into the human touch points of these patients in order to derive a detailed understanding of how the virus may have spread, and how and why it has genetically evolved. Our analysis provides a unique view to the dynamics of the 2019-nCoV coronavirus outbreak that complements knowledge obtained using other state-of-the-art methods such as Bayesian evolutionary analysis 9 .

\section{Materials and methods}

\section{Research data and preprocessing}

We obtained a total of 87 genomic sequences from human subjects that were available via the GISAID EpiFlu Database ${ }^{T M} 1$ as of Monday, February 10th 2020.

These data were preprocessed using the $\mathrm{R}$ programming language. First, we derived a metadata object that allows to store the sequence identifier, the collection location, the collection date, and the raw nucleotide sequence. The metadata object was then filtered to keep only those sequences for which the collection date was not empty and which featured a length that fell within a margin of 1,000 of the estimated 30,000 nucleotides that are currently assumed to make up the genomic code of the $2019-\mathrm{nCoV}$ coronavirus (i.e. we cover the range of nucleotide sequences from 29, 000 to 31,000 in length). This left us with a total of 82 genomic sequences. We then exported a single CSV file containing only the raw nucleotide sequences in ascending order by the collection date of the sample. This structure is the standard input format for the genes-CODON-samplesequence tokeniser featured in the Transcendental Information Cascades $\mathrm{R}$ toolchain that is available as free and open scientific software 2

\section{Construction of Transcendental Information Cascades}

Transcendental Information Cascades (TICs) are a method that first transforms any kind of sequential data into a network of recurring information tokens, to then exploit this network as a kind of analytical middle ground between views to the source data that would otherwise only be accessible individually through distinct analytical frameworks 16,17 . The approach falls into the category of contemporary approaches that make use of network theory for the study of dynamical complex systems [4, 19, 21]. In the field of phylogenetic and phylogeographic analysis our approach can be compared to haplotype networks for example [1, 18 but with the benefit that the resulting network preserves access to temporal patterns at both macroscopic (e.g. whole generation time periods) and microscopic (e.g. short time period bursts) resolution.

At an abstract level a Transcendental Information Cascade is constructed from some sequentially ordered source data in the following way: At first the data from each distinct sequence step is processed to extract unique information tokens. These tokens form the identifier set for that particular sequence step. Then a directed network is generated with one vertex for each sequence step and edges between any two vertices that have a particular

pos1\#+1\#AAG, pos1\#+2\#AGC, pos1\#+3\#GCT, pos $2 \#+1 \# C T C$, pos $2 \#+2 \# T C C$, pos $2 \#+3 \# C C G$, pos3\#+1\#CGA token from their identifier set in common and that does not occur in any identifier set of vertices that represent sequence steps between

1 https://www.gisaid.org/

2 https://github.com/vuw-c2lab/transcendental-information-cascades 
these two. In other words, identifier sets represent information token co-occurrences and edges represent information token recurrences.

As the information tokens of interest in this study we encode unique codon identifiers as (a) their position when sliding a window of size 3 in steps of size 3 over the nucleotide sequence (e.g. "pos1", "pos2", ...), (b) a flag that indicates the reading frame ("+1", " +2 " or " $+3 ")$ that captures the respective triplet in the current window, and (c) the actual matched nucleotide triplet that constitutes the matched codon. The use of this tokenisation is motivated by previous work on phylogenetic profiling and gene sequencing 6, 13 that suggested that codons suit well for assessing similarities (and dissimilarities) at the gene, chromosome, or genome levels 6, $6,24.22$.

An example of the particular encoding we use is visualised in Figure 1 . It is this tokenisation of the original nucleotide sequences that we use in the TIC approach to construct the information token recurrence network from. This means in our TIC instantiation the nodes represent distinct nucleotide sequences of the virus obtained from patient samples ordered by the date of their collection by researchers (random order in case of same date collections). The edges are unique codon identifier recurrences matched between different nucleotide sequences.

\section{Analysis of Transcendental Information Cascades}

The TIC network we derive in the way described before can be analysed in a variety of ways. Information-theoretic and network-theoretic measures have been introduced as the base framework to start the analysis from 16. In particular we perform (1) an analysis of the information token entropy and evenness over time, (2) an analysis of network clusters of sequences that can be detected in the TIC network, and (3) an analysis of the intra-cluster and inter-cluster nucleotide similarity of the original sequences.

Information token entropy and evenness We perform an analysis of information token entropy and information token evenness in order to understand whether the virus evolution can be considered an open or a closed system. Therefore we assess both measures in an accumulated fashion for each progression step through the ordered nucleotide sequences. In a closed system the information token entropy should strive towards an equilibrium of maximum entropy and not feature any wave-like up and down patterns. If we observe wave-like patterns it means nucleotide sequences at later progression stages feature codon identifiers that have not been observed in earlier nucleotide sequences.

So to measure information token entropy we assess which unique codon identifiers were seen up to a progression step and how often these were seen. This allows to compute the probability for every codon identifier, which then can be used to determine the information token entropy up to that point following the definition of Shannon entropy given as follows:

$$
H^{\prime}=-\sum_{i=1}^{R} p_{i} \ln p_{i}
$$

Analog to this we can then assess the information token evenness as per Pielou's species evenness index, which is defined as

$$
J^{\prime}=\frac{H^{\prime}}{H_{\max }^{\prime}}
$$

where $H^{\prime}$ is the measured Shannon entropy, and $H_{\max }^{\prime}$ is the maximum entropy that would be measured if all tokens would be equally likely to occur. 
Network analysis The Transcendental Information Cascade network we constructed can be mathematically and visually analysed in order to find structures that are of particular significance for a single epidemic outbreak or to compare different outbreaks structurally. In this study we focus on the former due to the lack of comparative data about other epidemic outbreaks.

We first perform a cluster analysis using the random walk apporach by Blondel et al. 3 on the weighted network that we can construct from our original TIC network by collapsing all edges between the same vertices to unique edges weighted by the sum of the collapsed edges (see Figure 2 for a schematic example and Figure 4 for the actual networks we construct following this approach). Afterwards, we analyse visually the network using the open source software Gephi. In Gephi we scale vertices by their degree and edges by their weight. Furthermore, we colour vertices by their cluster membership. We then run the Yifan $\mathrm{Hu} 12$ layout algorithm (optimal distance: 10,000; relative strength: 0.2 ; initial step size: 20 ; step ratio: 0.95 ; quadtree max level: 50 ; theta: 0.8 ; convergence threshold: $1 * 10^{-4}$; adaptive cooling: enabled) to visualise the network. We repeat the exact same process to construct and visualise three further networks (cf. Figures 5-7) that are representative of the TIC when exclusively focusing on the codon identifiers of each of the reading frames individually.
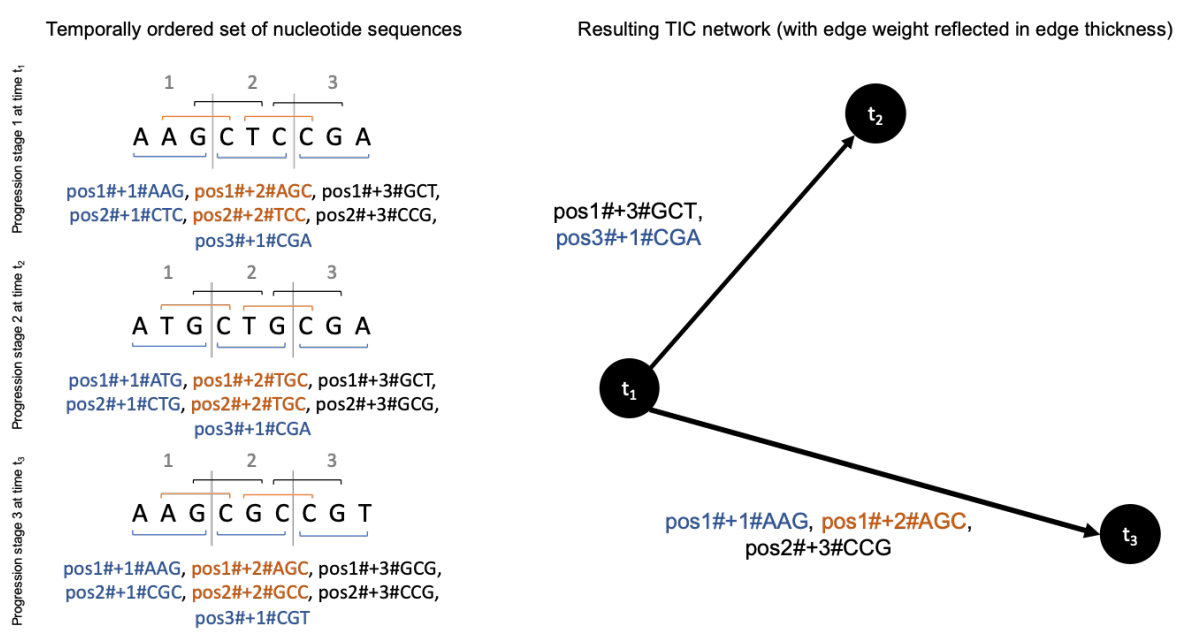

Figure 2. Schematic example of the weighted TIC network constructed from nucleotide sequences with tokenised unique codon identifiers.

Cluster evaluation In order to evaluate the meaningfulness of the TIC network against some baseline, we computer the intra-cluster and inter-cluster similarity of the raw nucleotide sequences. This is done by computing all pairwise comparisons of sequences within individual clusters (intra-cluster similarity) and comparison between all sequences from a cluster with all sequences that are not within that cluster (inter-cluster similarity) using the compareStrings function provided by the Biostrings $\mathrm{R}$ package 20 . The similarity of two sequences is then simply defined as follows:

$$
\operatorname{sim}(a, b)=1-\frac{\text { number of different nucleotides }}{\text { length of compared nucleotide sequence }}
$$

It is important to note that not all sequences we obtained had the exact same length (mean length of nucleotide sequences: 29861.95; standard deviation: 28.40705). Hence, we performed this comparison only on the sub-sequence from the first nucleotide position to the maximum nucleotide position of the shorter of the two sequences $a$ and $b$. 
Figure 3. Informationtheoretic assessment. Both entropy and evenness graphs feature wave-like patterns, indicating that the set of observed unique codon identifiers grows with increasing progression stages while the distribution of all observed unique codon identifiers remains rather even.

\section{Results and discussion}

\section{Information-theoretic analysis}

Figure 3 shows the graphs for the information token entropy and information token
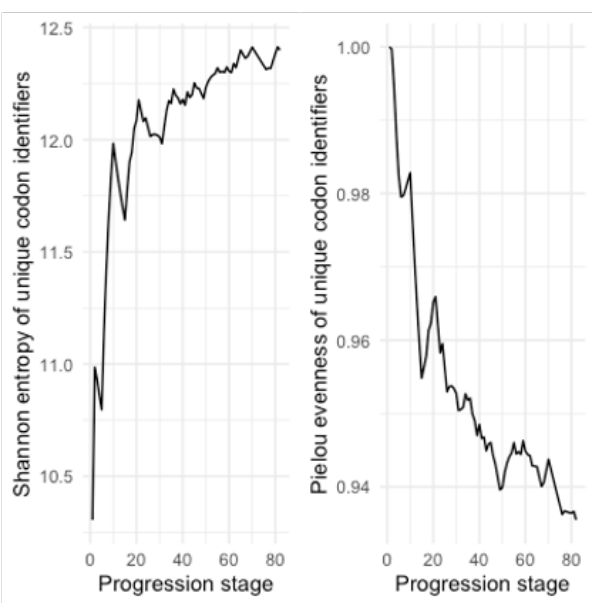
evenness. As one can see, these feature the aforementioned wave-like patterns, which means the system can be regarded open in the sense that over time the number of unique codon identifiers grows.

It is important to note that the evenness of the distribution of codon identifiers is relatively high, converging around a value of 0.95 . This means that those codon identifiers that have been observed are quite evenly distributed.

These results allow the interpretation that (a) there is evolving variation in the genomic sequences we studied, but (b) there is no indication for the dominance of a particular variance.

\section{Network analysis}

The weighted network derived from the original TIC network features a modularity of 0.762 and a diameter of 7 , which mainly reflects its small size and its particular structure that results from preserving temporal order in the sense that edges can only ever direct forward in time (i.e. to vertices that represent a later stage). However, with an average degree of 8.671 we find that there is certainly structure that differs from just simple step-by-step forward paths that lead to the consecutively next node. This latter result suggests that some codon paths branch and merge, emphasising that there is an evolving variation in the studied genomic sequences.

From the cluster analysis in combination with a visual inspection of the resulting graph (please refer to Figure 6 as well as Table 11 in the appendix) we find the following patterns that are noteworthy:

- At the center of the network one cluster emerges (dark red, cluster 0) that has only few stronger connections within the cluster but features connections to all other clusters and within itself through lower weighted edges. This cluster has no temporal focus, it comprises samples that were collected early as well as later during the outbreak. While the genome sequences that represent the beginning of the progression stage path in this cluster are from China, it then transitions geographically to Australia, Japan, Korea, Germany, Taiwan and Singapore.

- The light red cluster (cluster 1) comprises a coherent sequence of samples taken from patients in the original outbreak region (Wuhan) and is temporally focused on the early progression stages.

- The orange cluster starting from the bottom center of the network (cluster 2) is again first a sequence of samples from the original outbreak region in Wuhan, transitioning to Thailand (Nonthaburi), then back to China (Guangdong), before finishing in France.

- Cluster 3 (yellow) features samples taken in various places in China but also two later connections to the USA. 
- Cluster 4 (light green) is one of the smallest clusters but is spread fairly wide temporally. It features 4 samples taken in China and then transitions geographically to Singapore and France.

- In dark green (cluster 5) we can identify a cluster of genome data that was collected in Chonggqing, the USA and Singapore.

- The light blue cluster (cluster 6) is a temporally quite coherent sequence that links samples taken in Guangdong, Guangzhou and Foshan.

- A kind of international cluster (samples from Australia, the USA, England, Finland and Belgium) is represented by the dark blue cluster (cluster 7).

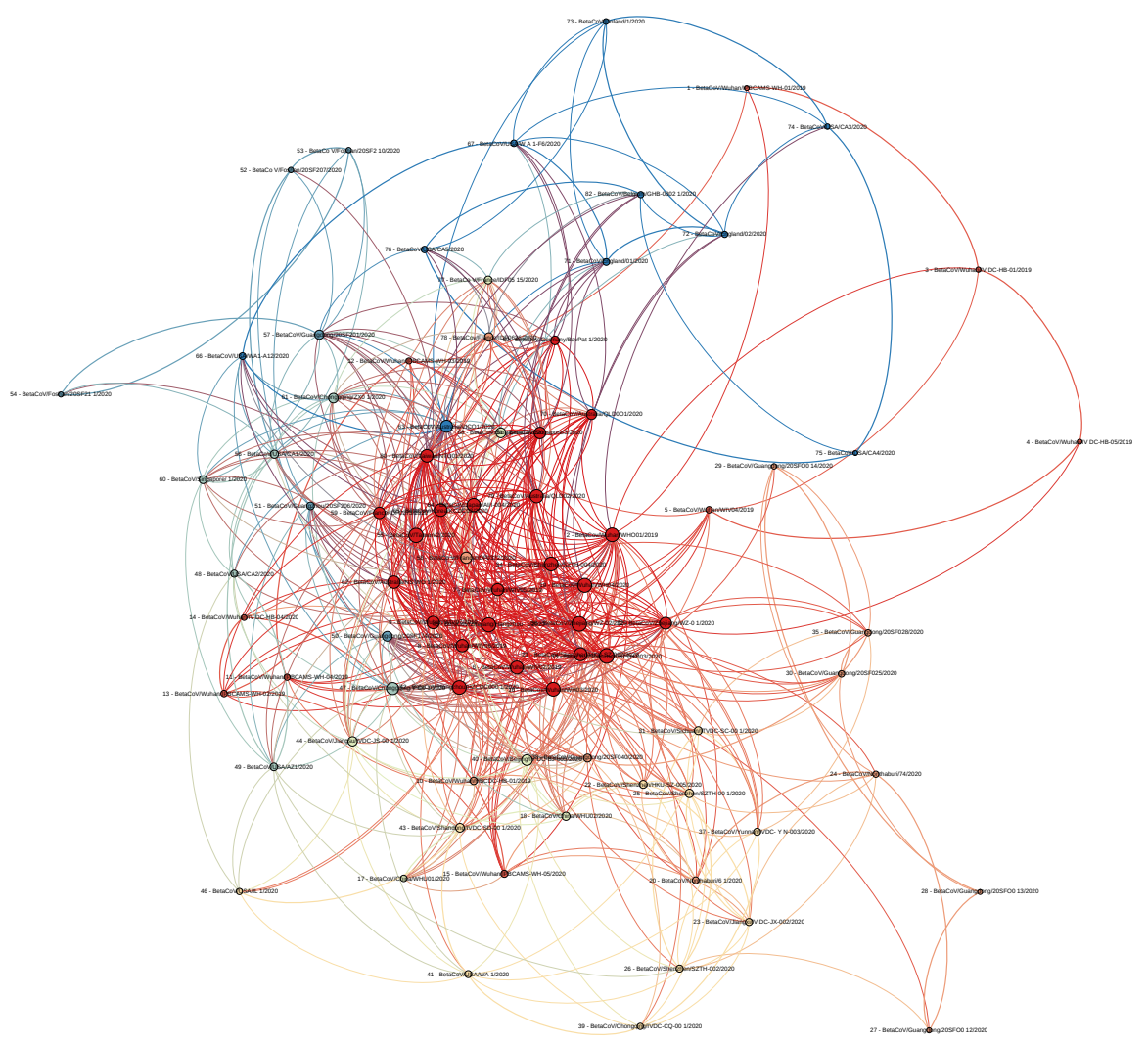

Figure 4. Figure 4. Weighted TIC network. From the original TIC network we generate a weighted network by collapsing all edges between the same vertices to unique edges weighted by the sum of the collapsed edges. The colours represent the nine identified network clusters. Node size represents the degree and edge thickness represents edge weight (in the sense that higher edge weight indicates a higher amount of codon identifiers shared by two vertices). Numeric indices before the genome identifiers represent the progression stage index of the particular vertex in the sequence of all ordered nucleotide sequences we analysed.

We suggest that while there are some obvious pathways of high similarity due to temporal order of the samples taken, there are still good indications for geographicaldependencies and transition phases that deserve further investigation. Transition points into and out of cluster 0 are particularly interesting in this regard, especially when the clusters linking in our out feature samples taken outside of China. We suggest that it 
may be worth to qualitatively assess the human-to-human connections the respective subjects had from which these samples were taken, and the places they have visited if there are no known links between the samples so far.

Triangulating the full TIC network with the networks that focus on individual reading frame similarity only (please refer to Figures 6, 77 and 8 as well as Tables 13,15 and 17 in the appendix) shows that the macroscopic structure is mostly consistent but much sparser in terms of low-weighted edges.

\section{Inter- and intra-cluster similarity}

From our measurement of the intra-cluster similarity (see Table 1) we find that the genomic similarity at the level of nucleotides is low in the central cluster 0 , which is expected because this is the largest of all clusters with low temporal coherence. However, cluster 5 also features a slightly lower average similarity with comparably high standard deviation, which indicates that this cluster may be slightly less coherent.

Table 1. Intra-cluster similarities.

\begin{tabular}{c|c|c|c|c|c} 
Cluster & Avg. similarity & SD & Min. similarity & Max. similarity & Cluster size \\
\hline \hline 0 & 0.277353 & 0.088247 & 0.250620 & 0.999866 & 24 \\
1 & 0.999730 & 0.000222 & 0.999231 & 1.000000 & 9 \\
2 & 0.999915 & 0.000048 & 0.999832 & 1.000000 & 11 \\
3 & 0.999385 & 0.000371 & 0.998461 & 0.999933 & 10 \\
4 & 0.998768 & 0.001345 & 0.996387 & 1.000000 & 6 \\
5 & 0.883701 & 0.112398 & 0.781976 & 0.999899 & 6 \\
6 & 0.999926 & 0.000036 & 0.999866 & 1.000000 & 6 \\
7 & 0.997900 & 0.002419 & 0.991158 & 1.000000 & 10
\end{tabular}

The barchart of the inter-cluster analysis results shown in Figure 5 reveals that there are some cluster pairs with high inter-cluster similarity, that the inter-cluster similarity is different between the +3 reading frame network and the other two reading frame networks, and that the pattern of the +3 reading frame network inter-cluster similarity is similar to the one for the entire TIC network. In particular we find that in the +1 and +2 reading frame networks the clusters 1, 4, 6 and 7 are quite similar at the nucleotide level compared to all other clusters that are pairwise distinct. For the entire TIC network and the +3 reading frame network instead we find that clusters $1,3,5$ and 7 show this kind of similarity. Altogether, these results indicate that (a) in some instances the clustering based on structural characteristics of the TIC network overrides actual genomic similarity, and (b) the choice of the reading frames during TIC construction has an impact on the resulting network that needs to be further investigated.

\section{Conclusion}

In this paper we presented a novel way to model, visualise and analyse the temporal dynamics of an epidemic outbreak. We applied Transcendental Information Cascades to capture the recurrence and co-occurrence of unique codon identifiers over distinct and temporally ordered nucleotide sequences derived from genetic samples taken from different human subjects. Our case study was focused on the recent outbreak of the novel 2019-nCoV coronavirus. We find clusters and evolution pathways of virus genomes in the constructed Transcendental Information Cascade that are candidates to be investigated further for potential human-to-human transmission paths and genetic evolution of the 2019-nCoV coronavirus.

Our study was performed as a snapshot case study on a limited sample of virus data. While this was at the time of this study all data that was available, we will need to re-execute this analysis continuously as more data becomes available to check whether any of our results change. Furthermore, our results indicate that experiments with 
(a)
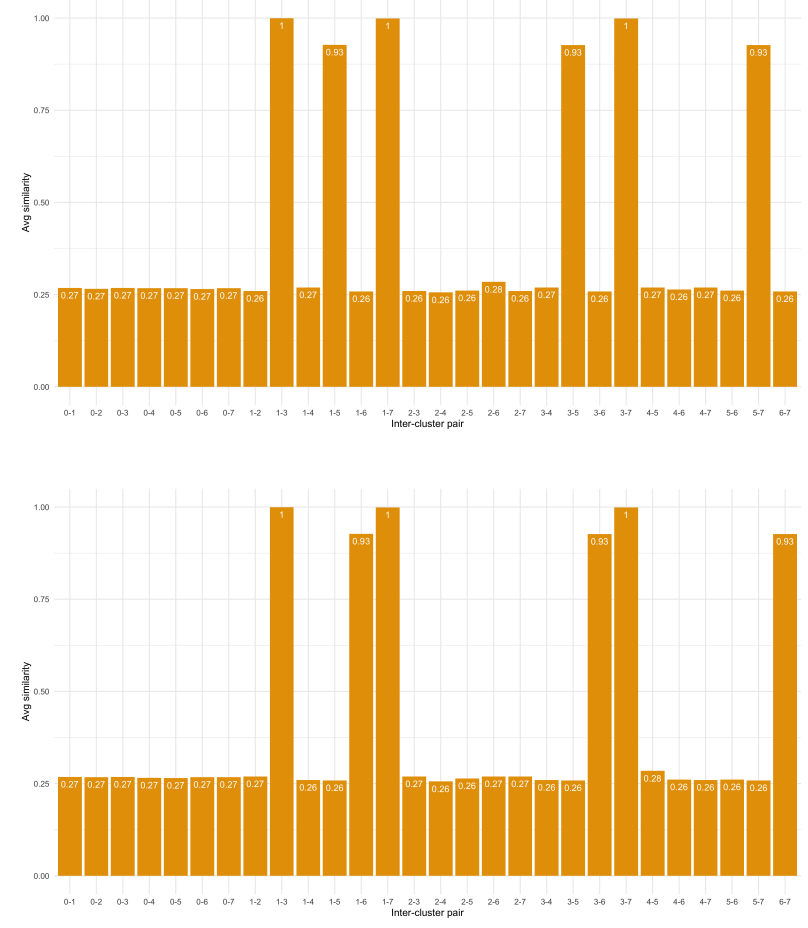

(b)

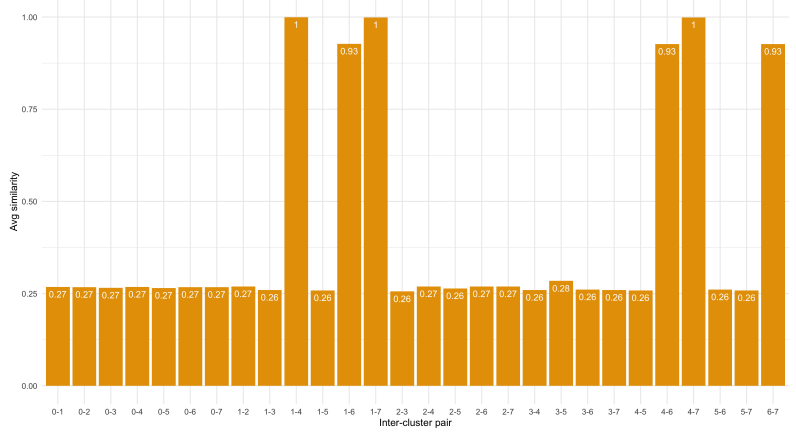

(c)

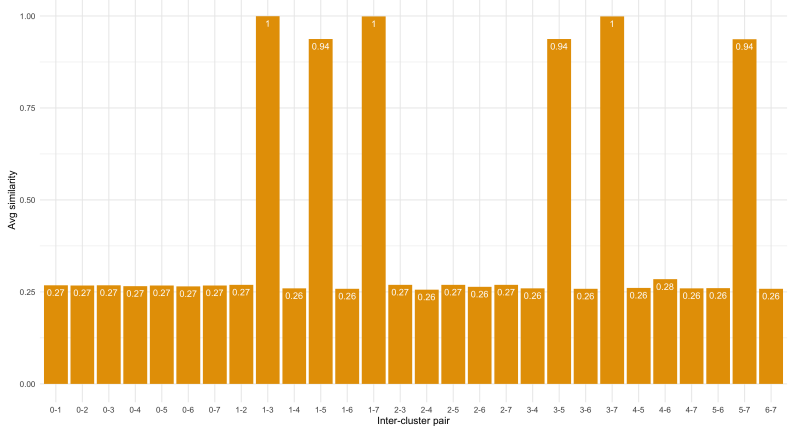

(d)

Figure 5. Overview of inter-cluster similarities for (a) the full TIC network, (b) the +1 reading frame network, (c) the +2 reading frame network, and (d) the +3 reading frame network. 
alternative tokenisations are needed, such as weighting the $+1,+2$ and +3 reading frames differently (or omitting certain frames entirely) depending on their functional role $[10]$. We also suggest that it will be important to perform a wider variety of analysis of the generated TIC data (e.g. testing of different network clustering methods, application of dynamical systems analyses techniques) to get more profound insights. Our analysis also does not take measurement biases into account that may results from different techniques used by different laboratories for genome extraction and analysis 7 .

In summary, the approach presented in this paper is exploratory and hypotheses generating in nature. It does not confirm or reject any of the hypotheses we generated about the potential transmission and evolution pathways nor does it provide causal explanation for the patterns found. However, the purpose of our work is to provide an alternative view to the epidemic dynamics of the 2019-nCoV outbreak (and potential other outbreaks in the future) that is hidden to state-of-the-art methods, and that may trigger geneticists and epidemiologists to look at pathways in epidemic outbreaks they would not normally be aware of.

\section{References}

1. H.-J. Bandelt, P. Forster, and A. Röhl. Median-joining networks for inferring intraspecific phylogenies. Molecular biology and evolution, 16(1):37-48, 1999.

2. D. Benvenuto, M. Giovannetti, A. Ciccozzi, S. Spoto, S. Angeletti, and M. Ciccozzi. The 2019-new coronavirus epidemic: evidence for virus evolution. Journal of Medical Virology, 2020.

3. V. D. Blondel, J.-L. Guillaume, R. Lambiotte, and E. Lefebvre. Fast unfolding of communities in large networks. Journal of statistical mechanics: theory and experiment, 2008(10):P10008, 2008.

4. S. Boccaletti, G. Bianconi, R. Criado, C. I. Del Genio, J. Gómez-Gardenes, M. Romance, I. Sendina-Nadal, Z. Wang, and M. Zanin. The structure and dynamics of multilayer networks. Physics Reports, 544(1):1-122, 2014.

5. O. Cenciarelli, S. Pietropaoli, A. Malizia, M. Carestia, F. D'Amico, A. Sassolini, D. Di Giovanni, S. Rea, V. Gabbarini, A. Tamburrini, et al. Ebola virus disease 2013-2014 outbreak in west africa: an analysis of the epidemic spread and response. International journal of microbiology, 2015, 2015.

6. P. Cliften, P. Sudarsanam, A. Desikan, L. Fulton, B. Fulton, J. Majors, R. Waterston, B. A. Cohen, and M. Johnston. Finding functional features in saccharomyces genomes by phylogenetic footprinting. science, 301(5629):71-76, 2003.

7. A. S. Devonshire, A. S. Whale, A. Gutteridge, G. Jones, S. Cowen, C. A. Foy, and J. F. Huggett. Towards standardisation of cell-free dna measurement in plasma: controls for extraction efficiency, fragment size bias and quantification. Analytical and bioanalytical chemistry, 406(26):6499-6512, 2014.

8. N. Dong, X. Yang, L. Ye, K. Chen, E. W.-C. Chan, M. Yang, and S. Chen. Genomic and protein structure modelling analysis depicts the origin and infectivity of 2019ncov, a new coronavirus which caused a pneumonia outbreak in wuhan, china. bioRxiv, 2020.

9. A. J. Drummond and A. Rambaut. Beast: Bayesian evolutionary analysis by sampling trees. BMC evolutionary biology, 7(1):214, 2007. 
10. P. J. Farabaugh and G. R. Björk. How translational accuracy influences reading frame maintenance. The EMBO journal, 18(6):1427-1434, 1999.

11. J. Faulkner, M. Schaller, J. H. Park, and L. A. Duncan. Evolved disease-avoidance mechanisms and contemporary xenophobic attitudes. Group Processes 83 Intergroup Relations, 7(4):333-353, 2004.

12. Y. Hu. Efficient, high-quality force-directed graph drawing. Mathematica Journal, 10(1):37-71, 2005.

13. M. Kellis, N. Patterson, M. Endrizzi, B. Birren, and E. S. Lander. Sequencing and comparison of yeast species to identify genes and regulatory elements. Nature, 423(6937):241, 2003.

14. C. Li, X. Yu, and N. Helal. Similarity analysis of dna sequences based on codon usage. Chemical physics letters, 459(1-6):172-174, 2008.

15. T. Liu, J. Hu, M. Kang, L. Lin, H. Zhong, J. Xiao, G. He, T. Song, Q. Huang, Z. Rong, et al. Transmission dynamics of 2019 novel coronavirus (2019-ncov). bioRxiv, 2020.

16. M. Luczak-Roesch, K. O'Hara, J. D. Dinneen, and R. Tinati. What an entangled web we weave: An information-centric approach to time-evolving socio-technical systems. Minds and Machines, 28(4):709-733, 2018.

17. M. Luczak-Roesch, R. Tinati, M. Van Kleek, and N. Shadbolt. From coincidence to purposeful flow? properties of transcendental information cascades. In 2015 IEEE/ACM International Conference on Advances in Social Networks Analysis and Mining (ASONAM), pages 633-638. IEEE, 2015.

18. I. Manolopoulou, L. Legarreta, B. C. Emerson, S. Brooks, and S. Tavaré. A bayesian approach to phylogeographic clustering. Interface focus, 1(6):909-921, 2011.

19. N. Masuda and P. Holme. Detecting sequences of system states in temporal networks. Scientific reports, 9(1):1-11, 2019.

20. G. R. D. S. Pagès H, Aboyoun P. Biostrings: Efficient manipulation of biological strings, 2019. R package version 2.54.0.

21. M. A. Porter. Nonlinearity+ networks: A 2020 vision. arXiv preprint arXiv:1911.03805, 2019.

22. P. M. Sharp and W.-H. Li. The codon adaptation index-a measure of directional synonymous codon usage bias, and its potential applications. Nucleic acids research, 15(3):1281-1295, 1987.

23. R. D. Smith. Responding to global infectious disease outbreaks: lessons from sars on the role of risk perception, communication and management. Social science $\mathbb{E}$ medicine, 63(12):3113-3123, 2006. 


\section{Appendix}

The software developed for this particular analysis is part of the open scientific software "R toolchain to construct and analyse Transcendental Information Cascades" that is available via https://github.com/vuw-c2lab/transcendental-information-cascades/ tree/master/input/2020-02-03-2019nCoV. For reproduction purposes it is necessary to obtain the raw genomic data used in this study from the GISAID EpiFlu Database ${ }^{T M}$.

For further reference we provide the raw results of some of our analysis. We particularly point to the tables that provide references for relationships between nucleotide sequences and the cluster membership of nucleotide sequences. 
Table 2. Edge weight between distinct nucleotide sequences in the Transcendental Information Cascade we constructed.

\begin{tabular}{|c|c|c|}
\hline Source sequence & Target sequence & Edge weight \\
\hline 11 - BetaCoV/Wuhan/IPBCAMS-WH-04/2019 & (12-BetaCoV/Wuhan/IPBCAMS-WH-03/2019 & 29885 \\
\hline $3-\mathrm{BetaCoV} /$ Wuhan/IV DC-HB-01/2019 & $4-\mathrm{BetaCoV} /$ Wuhan/IV DC-HB-05/2019 & 29883 \\
\hline 4- BetaCoV/Wuhan/IV DC-HB-05/2019 & 5 - BetaCoV/Wuhan/WIV04/2019 & 29883 \\
\hline $66-\mathrm{BetaCoV} / \mathrm{USA} / \mathrm{WA} 1-\mathrm{A} 12 / 2020$ & $67-\mathrm{BetaCoV} / \mathrm{USA} / \mathrm{W}$ A $1-\mathrm{F} 6 / 2020$ & 29880 \\
\hline $74-\mathrm{BetaCoV} / \mathrm{USA} / \mathrm{CA} 3 / 2020$ & $75-\mathrm{BetaCoV} / \mathrm{USA} / \mathrm{CA} 4 / 2020$ & 29880 \\
\hline 17 - BetaCoV/China/WHU01/2020 & 18 - BetaCoV/China/WHU02/2020 & 29879 \\
\hline $14-\mathrm{BetaCoV} /$ Wuhan/IV DC-HB-04/2020 & $15-$ BetaCoV/Wuhan/IPBCAMS-WH-05/2020 & \\
\hline 12 - BetaCoV/Wuhan/IPBCAMS-WH-03/2019 & 13 - BetaCoV/Wuhan/IPBCAMS-WH-02/2019 & 29870 \\
\hline 13 - BetaCoV/Wuhan/IPBCAMS-WH-02/2019 & $14-$ BetaCoV/Wuhan/IV DC-HB-04/2020 & 29867 \\
\hline $75-\mathrm{BetaCoV} / \mathrm{USA} / \mathrm{CA} 4 / 2020$ & $76-\mathrm{BetaCoV} / \mathrm{USA} / \mathrm{CA} 5 / 2020$ & 29865 \\
\hline $48-\mathrm{BetaCoV} / \mathrm{USA} / \mathrm{CA} 2 / 2020$ & $49-\mathrm{BetaCoV} / \mathrm{USA} / \mathrm{AZ} 1 / 2020$ & 29862 \\
\hline $22-$ BetaCoV/Shenzhen/HKU-SZ-005/2020 & 23 - BetaCoV/Jiangxi/IV DC-JX-002/2020 & 29856 \\
\hline $27-$ BetaCoV/Guangdong/20SFO0 $12 / 2020$ & $28-$ BetaCoV/Guangdong/20SFO0 13/2020 & 29843 \\
\hline $28-$ BetaCoV/Guangdong/20SFOO $13 / 2020$ & $29-$ BetaCoV/Guangdong/20SFO0 14/2020 & 29831 \\
\hline $29-$ BetaCoV/Guangdong/20SFO0 14/2020 & $30-$ BetaCoV/Guangdong/20SF025/2020 & 29831 \\
\hline $71-$ BetaCoV/England/01/2020 & $72-$ BetaCoV/England/02/2020 & 29830 \\
\hline 52 - BetaCo V/Foshan/20SF 207/2020 & 53 - BetaCo V/Foshan/20SF2 10/2020 & 29828 \\
\hline $51-$ BetaCoV/Guangzhou/20SF206/2020 & $52-$ BetaCo V/Foshan/20SF207/2020 & 29825 \\
\hline $25-$ BetaCoV/Shenzhen/SZTH-00 $1 / 2020$ & $26-$ BetaCoV/Shenzhen/SZTH-002/2020 & 29813 \\
\hline $53-$ BetaCo V/Foshan/20SF2 10/2020 & $54-\mathrm{BetaCoV} /$ Foshan/20SF21 1/2020 & 29801 \\
\hline $50-$ BetaCoV/Guangdong/20SF174/2020 & $51-$ BetaCoV/Guangzhou/20SF 206/2020 & 29797 \\
\hline 73 - BetaCoV/Finland/1/2020 & $74-\mathrm{BetaCoV} / \mathrm{USA} / \mathrm{CA} 3 / 2020$ & 29665 \\
\hline 72 - BetaCoV/England/02/2020 & $73-$ BetaCoV/Finland $/ 1 / 2020$ & 29643 \\
\hline $1-$ BetaCoV/Wuhan/IPBCAMS-WH-01/2019 & 3-BetaCoV/Wuhan/IV DC-HB-01/2019 & 29346 \\
\hline $37-$ BetaCoV/Yunnan/IVDC- Y N-003/2020 & 39 - BetaCoV/Chongqing/IVDC-CQ-00 1/2020 & 29332 \\
\hline $26-$ BetaCoV/Shenzhen/SZTH-002/2020 & $31-$ BetaCoV/Sichuan/ITVDC-SC-00 1/2020 & 29315 \\
\hline $24-$ BetaCoV/Nonthaburi/ $74 / 2020$ & $27-$ BetaCoV/Guangdong/20SFO0 12/2020 & 29290 \\
\hline 39 - BetaCoV/Chongqing/IVDC-CQ-00 1/2020 & $41-\mathrm{BetaCoV} /$ USA/WA $1 / 2020$ & 29274 \\
\hline 23 - BetaCoV/Jiangxi/IV DC-JX-002/2020 & $25-$ BetaCoV/Shenzhen/SZTH-00 1/2020 & 29248 \\
\hline $41-\mathrm{BetaCoV} / \mathrm{USA} /$ WA $1 / 2020$ & $43-$ BetaCoV/Shandong/IVDC-SD-00 $1 / 2020$ & 29142 \\
\hline $54-$ BetaCoV/Foshan/20SF $21 \quad 1 / 2020$ & $57-$ BetaCoV/Guangdong/20SF 201/2020 & 28741 \\
\hline $49-\mathrm{BetaCoV} / \mathrm{USA} / \mathrm{AZ} 1 / 2020$ & 56 - BetaCoV/USA/CA1/2020 & 28651 \\
\hline $40-$ BetaCoV/Beijing/IV DC-BJ-005/2020 & $44-$ BetaCoV/Jiangsu/IVDC-JS-00 1/2020 & 28650 \\
\hline $20-\mathrm{BetaCoV} /$ Nonthaburi/6 $1 / 2020$ & $24-\mathrm{BetaCoV} /$ Nonthaburi/74/2020 & 28586 \\
\hline 63 - BetaCoV/Australia/VICO1/2020 & $66-\mathrm{BetaCoV} / \mathrm{USA} / \mathrm{WA} 1-\mathrm{A} 12 / 2020$ & 28557 \\
\hline 35 - BetaCoV/Guangdong/20SF028/2020 & 38 - BetaCoV/Guangdong/20SF040/2020 & 28532 \\
\hline 43 - BetaCoV/Shandong/IVDC-SD-00 1/2020 & $46-\mathrm{BetaCoV} / \mathrm{USA} / \mathrm{IL} 1 / 2020$ & 28469 \\
\hline $56-$ BetaCoV/USA/CA1/2020 & $60-$ BetaCoV/Singapore/ $1 / 2020$ & 28287 \\
\hline 65 - BetaCoV/Korea/KCDC03/2020 & $70-$ BetaCoV/Australia/QLDOO1/2020 & 28136 \\
\hline 68 - BetaCoV/Singapore/2/2020 & 77 - BetaCo V/France/IDF05 15/2020 & 28051 \\
\hline $30-$ BetaCoV/Guangdong/20SF025/2020 & 35 - BetaCoV/Guangdong/20SF028/2020 & 27962 \\
\hline $67-\mathrm{BetaCoV} / \mathrm{USA} / \mathrm{W}$ A $1-\mathrm{F} 6 / 2020$ & $71-$ BetaCoV/England/01/2020 & 27952 \\
\hline 31 - BetaCoV/Sichuan/ITVDC-SC-00 1/2020 & $37-$ BetaCoV/Yunnan/IVDC- Y N-003/2020 & 27711 \\
\hline $10-$ BetaCoV/Wuhan/HBCDC-HB-01/2019 & $20-$ BetaCoV/Nonthaburi/6 1/2020 & 27513 \\
\hline 62 - BetaCoV/Australia/NS WO $1 / 2020$ & $69-$ BetaCoV/Germany/BavPat $1 / 2020$ & 27286 \\
\hline 76 - BetaCoV/USA/CA5/2020 & $82-$ BetaCoV/Belgium/GHB-0302 1/2020 & 27215 \\
\hline 15 - BetaCoV/Wuhan/IPBCAMS-WH-05/2020 & $22-\mathrm{BetaCoV} /$ Shenzhen/HKU-SZ-005/2020 & 27049 \\
\hline $5-\mathrm{BetaCoV} /$ Wuhan/WIV04/2019 & 11 - BetaCoV/Wuhan/IPBCAMS-WH-04/2019 & 26905 \\
\hline 38 - BetaCoV/Guangdong/20SF040/2020 & 58 - BetaCo V/France/IDF0372/2020 & 26795 \\
\hline 58 - BetaCo V/France/IDF0372/2020 & 78 - BetaCov/France/IDF0626/2020 & 26624 \\
\hline 18 - BetaCoV/China/WHU02/2020 & $40-$ BetaCoV/Beijing/IV DC-BJ-005/2020 & 25949 \\
\hline 32 - BetaCoV/Zhejiang/WZ-0 $1 / 2020$ & $59-$ BetaCoV/France/IDF0373/2020 & 25427 \\
\hline $44-$ BetaCoV/Jiangsu/IVDC-JS-00 $1 / 2020$ & $68-$ BetaCoV/Singapore/2/2020 & 22332 \\
\hline $60-$ BetaCoV/Singapore/ $1 / 2020$ & $61-$ BetaCoV/Chonggqing/ZXo 1/2020 & 21150 \\
\hline $47-$ BetaCoV/Chonggqing/Y CO $1 / 2020$ & $48-\mathrm{BetaCoV} / \mathrm{USA} / \mathrm{CA} 2 / 2020$ & 21147 \\
\hline $46-\mathrm{BetaCoV} / \mathrm{USA} / \mathrm{IL} 1 / 2020$ & $47-$ BetaCoV/Chonggqing/Y CO $1 / 2020$ & 21143 \\
\hline 61 - BetaCoV/Chonggqing/ZXo $1 / 2020$ & $63-$ BetaCoV/Australia/VICO1/2020 & 20532 \\
\hline 45 - BetaCoV/Zhejiang/Hangzhou- $1 / 2020$ & $55-$ BetaCoV/Taiwan $/ 2 / 2020$ & 20288 \\
\hline $33-$ BetaCoV/Shenzhen/SZTH-003/2020 & $34-$ BetaCoV/Shenzhen/SZTH-004/2020 & 20102 \\
\hline $46-\mathrm{BetaCoV} / \mathrm{USA} / \mathrm{IL} 1 / 2020$ & $48-\mathrm{BetaCoV} / \mathrm{USA} / \mathrm{CA} 2 / 2020$ & 8710 \\
\hline 60 - BetaCoV/Singapore/ $1 / 2020$ & 63 - BetaCoV/Australia/VICO1/2020 & 8389 \\
\hline $47-$ BetaCoV/Chonggqing/Y CO $1 / 2020$ & $61-$ BetaCoV/Chongging/ZXo $1 / 2020$ & 7936 \\
\hline 55 - BetaCoV/Taiwan/2/2020 & $81-\mathrm{BetaCoV} /$ Singapore/3/2020 & 4399 \\
\hline 55 - BetaCoV/Taiwan/2/2020 & 68 - BetaCoV/Singapore/2/2020 & 3424 \\
\hline $44-$ BetaCoV/Jiangsu/IVDC-JS-00 $1 / 2020$ & 55 - BetaCoV/Taiwan/2/2020 & $\begin{array}{l}3424 \\
3393\end{array}$ \\
\hline $55-$ BetaCoV/Taiwan/2/2020 & $64-\mathrm{BetaCoV} / \mathrm{Japan} / \mathrm{AI} / \mathrm{I}-004 / 2020$ & 904 \\
\hline $5-$ BetaCoV/Wuhan/WIV04/2019 & 6-BetaCoV/Wuhan/WIV02/2019 & 848 \\
\hline 62 - BetaCoV/Australia/NS WO $1 / 2020$ & $63-$ BetaCoV/Australia/VICO1/2020 & 838 \\
\hline 69 - BetaCoV/Germany/BavPat $1 / 2020$ & $71-$ BetaCoV/England/01/2020 & 826 \\
\hline $67-\mathrm{BetaCoV} / \mathrm{USA} / \mathrm{W}$ A $1-\mathrm{F} 6 / 2020$ & $69-$ BetaCoV/Germany/BavPat 1/2020 & 815 \\
\hline $10-\mathrm{BetaCoV} /$ Wuhan/HBCDC-HB-01/2019 & $16-$ BetaCov/Wuhan/WHO3/2020 & 812 \\
\hline 16 - BetaCov/Wuhan/WHO3/2020 & 20 - BetaCoV/Nonthaburi $/ 61 / 2020$ & 806 \\
\hline 78 - BetaCov/France/IDF0626/2020 & $81-$ BetaCoV/Singapore/3/2020 & 779 \\
\hline $61-$ BetaCoV/Chonggqing/ZXo 1/2020 & 62 - BetaCoV/Australia/NS WO 1/2020 & 773 \\
\hline $59-$ BetaCoV/France/IDF0373/2020 & $65-$ BetaCoV/Korea/KCDC03/2020 & 773 \\
\hline $6-\mathrm{BetaCoV} /$ Wuhan/WIV02/2019 & $11-\mathrm{BetaCoV} / \mathrm{Wuhan} / \mathrm{IPBCAMS}-\mathrm{WH}-04 / 2019$ & 771 \\
\hline 35 - BetaCoV/Guangdong/20SF028/2020 & $36-$ BetaCoV/Zhejiang/WZ-02/2020 & 771 \\
\hline $36-$ BetaCoV/Zhejiang/WZ-02/2020 & 38 - BetaCoV/Guangdong/20SF040/2020 & \\
\hline 45- BetaCoV/Zhejiang/Hangzhou- $1 / 2$ & $46-\mathrm{BetaCoV} / \mathrm{USA} / \mathrm{IL} 1 / 2020$ & 762 \\
\hline 78 - BetaCov/France/IDF0626/2020 & $80-$ BetaCoV/Taiwan/NTU01/2020 & 758 \\
\hline $8-\mathrm{BetaCoV} /$ Wuhan/WIV06/2019 & $10-$ BetaCoV/Wuhan/HBCDC-HB-01/2019 & 755 \\
\hline
\end{tabular}


Table 3. Edge weight between distinct nucleotide sequences in the Transcendental Information Cascade we constructed (contd.).

\begin{tabular}{|c|c|c|}
\hline Source sequence & Target sequence & Edge weight \\
\hline 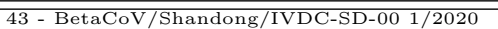 & "45- BetaCoV/Zhejiang/Hangzhou- $1 / 2020$ & $\overline{7754}$ \\
\hline 19 - BetaCov/Wuhan/WH04/2020 & $22-$ BetaCoV/Shenzhen/HKU-SZ-005/2020 & 748 \\
\hline $34-$ BetaCoV/Shenzhen/SZTH-004/2020 & 36 - BetaCoV/Zhejiang/WZ-02/2020 & 747 \\
\hline 57 - BetaCoV/Guangdong/20SF 201/2020 & 58 - BetaCo V/France/IDF0372/2020 & 742 \\
\hline $69-$ BetaCoV/Germany/BavPat 1/2020 & 79 - BetaCoV/Australia/QLD02/2020 & 740 \\
\hline $15-\mathrm{BetaCoV} /$ Wuhan/IPBCAMS-WH-05/2020 & $19-$ BetaCov/Wuhan/WH04/2020 & 731 \\
\hline $20-\mathrm{BetaCoV} /$ Nonthaburi/6 1/2020 & $21-$ BetaCoV/Shenzhen/HKU-SZ-002/2020 & 727 \\
\hline $6-\mathrm{BetaCoV} /$ Wuhan/WIV02/2019 & $7-\mathrm{BetaCoV} /$ Wuhan/WIV05/2019 & 725 \\
\hline $21-$ BetaCoV/Shenzhen/HKU-SZ-002/2020 & $24-$ BetaCoV/Nonthaburi $/ 74 / 2020$ & 715 \\
\hline $80-$ BetaCoV/Taiwan/NTU01/2020 & $81-$ BetaCoV/Singapore/3/2020 & 714 \\
\hline $9-$ BetaCoV/Wuhan/WIV07/2019 & $17-$ BetaCoV/China/WHU01/2020 & 713 \\
\hline 5 - BetaCoV/Wuhan/WIV04/2019 & $7-$ BetaCoV/Wuhan/WIV05/2019 & 711 \\
\hline 7 - BetaCoV/Wuhan/WIV05/2019 & $19-$ BetaCov/Wuhan/WH04/2020 & 703 \\
\hline $76-\mathrm{BetaCoV} / \mathrm{USA} / \mathrm{CA} 5 / 2020$ & 79 - BetaCoV/Australia/QLD02/2020 & 700 \\
\hline 79 - BetaCoV/Australia/QLD02/2020 & $82-$ BetaCoV/Belgium/GHB-0302 1/2020 & 700 \\
\hline 7 - BetaCoV/Wuhan/WIV05/2019 & $11-$ BetaCoV/Wuhan/IPBCAMS-WH-04/2019 & 698 \\
\hline $41-\mathrm{BetaCoV} / \mathrm{USA} / \mathrm{WA} 1 / 2020$ & $42-$ BetaCoV/Hangzhou/HZCDC000 $1 / 2020$ & 698 \\
\hline $42-$ BetaCoV/Hangzhou/HZCDC000 1/2020 & $43-$ BetaCoV/Shandong/IVDC-SD-00 1/2020 & 698 \\
\hline 63 - BetaCoV/Australia/VICO1/2020 & $64-\mathrm{BetaCoV} / J a p a n / A I / I-004 / 2020$ & 694 \\
\hline 38 - BetaCoV/Guangdong/20SF040/2020 & 50 - BetaCoV/Guangdong/20SF 174/2020 & 693 \\
\hline $68-$ BetaCoV/Singapore/2/2020 & 70 - BetaCoV/Australia/QLD0O1/2020 & 687 \\
\hline $2-$ BetaCov/Wuhan/WHO01/2019 & $9-$ BetaCoV/Wuhan/WIV07/2019 & 686 \\
\hline $21-$ BetaCoV/Shenzhen/HKU-SZ-002/2020 & 33 - BetaCoV/Shenzhen/SZTH-003/2020 & 685 \\
\hline $21-$ BetaCoV/Shenzhen/HKU-SZ-002/2020 & $50-$ BetaCoV/Guangdong/20SF $174 / 2020$ & 685 \\
\hline $55-$ BetaCoV/Taiwan $/ 2 / 2020$ & 56 - BetaCoV/USA/CA1/2020 & 685 \\
\hline $36-$ BetaCoV/Zhejiang/WZ-02/2020 & 50 - BetaCoV/Guangdong/20SF174/2020 & 678 \\
\hline $55-$ BetaCoV/Taiwan $/ 2 / 2020$ & $62-$ BetaCoV/Australia/NS WO $1 / 2020$ & 677 \\
\hline $49-\mathrm{BetaCoV} / \mathrm{USA} / \mathrm{AZ} 1 / 2020$ & 55 - BetaCoV/Taiwan/2/2020 & 676 \\
\hline $65-\mathrm{BetaCoV} /$ Korea/KCDC03/2020 & 68 - BetaCoV/Singapore/2/2020 & 675 \\
\hline 70 - BetaCoV/Australia/QLD0O1/2020 & 77 - BetaCo V/France/IDF05 15/2020 & 675 \\
\hline 16 - BetaCov/Wuhan/WH03/2020 & $21-$ BetaCoV/Shenzhen/HKU-SZ-002/2020 & 674 \\
\hline $64-\mathrm{BetaCoV} / \mathrm{Japan} / \mathrm{AI} / \mathrm{I}-004 / 2020$ & $66-\mathrm{BetaCoV/USA} / \mathrm{WA} 1-\mathrm{A} 12 / 2020$ & 674 \\
\hline 62 - BetaCoV/Australia/NS WO $1 / 2020$ & $64-\mathrm{BetaCoV} / \mathrm{Japan} / \mathrm{AI} / \mathrm{I}-004 / 2020$ & 671 \\
\hline $19-$ BetaCov/Wuhan/WHO4/2020 & 42 - BetaCoV/Hangzhou/HZCDC000 1/2020 & 666 \\
\hline $42-$ BetaCoV/Hangzhou/HZCDC000 1/2020 & $45-$ BetaCoV/Zhejiang/Hangzhou- $1 / 2020$ & 665 \\
\hline 8 - BetaCoV/Wuhan/WIV06/2019 & 16 - BetaCov/Wuhan/WH03/2020 & 664 \\
\hline $21-$ BetaCoV/Shenzhen/HKU-SZ-002/2020 & 36 - BetaCoV/Zhejiang/WZ-02/2020 & 654 \\
\hline $42-$ BetaCoV/Hangzhou/HZCDC000 $1 / 2020$ & $64-$ BetaCoV/Japan/AI/I-004/2020 & 654 \\
\hline $9-\mathrm{BetaCoV} /$ Wuhan/WIV07/2019 & $32-$ BetaCoV/Zhejiang/WZ-0 1/2020 & 651 \\
\hline 30 - BetaCoV/Guangdong/20SF025/2020 & 33 - BetaCoV/Shenzhen/SZTH-003/2020 & 646 \\
\hline $18-$ BetaCoV/China/WHU02/2020 & $32-$ BetaCoV/Zhejiang/WZ-0 1/2020 & 641 \\
\hline $64-$ BetaCoV/Japan/AI/I-004/2020 & $69-$ BetaCoV/Germany/BavPat $1 / 2020$ & 637 \\
\hline $42-$ BetaCoV/Hangzhou/HZCDC000 1/2020 & $62-$ BetaCoV/Australia/NS WO $1 / 2020$ & 630 \\
\hline $32-$ BetaCoV/Zhejiang/WZ-0 $1 / 2020$ & $40-$ BetaCoV/Beijing/IV DC-BJ-005/2020 & 629 \\
\hline $34-\mathrm{BetaCoV} /$ Shenzhen/SZTH-004/2020 & 35 - BetaCoV/Guangdong/20SF028/2020 & 628 \\
\hline 7 - BetaCoV/Wuhan/WIV05/2019 & 45 - BetaCoV/Zhejiang/Hangzhou- $1 / 2020$ & 625 \\
\hline $64-\mathrm{BetaCoV} / \mathrm{Japan} / \mathrm{AI} / \mathrm{I}-004 / 2020$ & 79 - BetaCoV/Australia/QLD02/2020 & 619 \\
\hline $19-$ BetaCov/Wuhan/WH04/2020 & $45-$ BetaCoV/Zhejiang/Hangzhou- 1/2020 & 618 \\
\hline $8-$ BetaCoV/Wuhan/WIV06/2019 & $50-$ BetaCoV/Guangdong/20SF $174 / 2020$ & 616 \\
\hline 2- BetaCov/Wuhan/WHO01/2019 & 17 - BetaCoV/China/WHU01/2020 & 613 \\
\hline $43-\mathrm{BetaCoV} /$ Shandong/IVDC-SD-00 $1 / 2020$ & $44-$ BetaCoV/Jiangsu/IVDC-JS-00 $1 / 2020$ & 608 \\
\hline $57-$ BetaCoV/Guangdong/20SF 201/2020 & 80 - BetaCoV/Taiwan/NTU01/2020 & 607 \\
\hline 16 - BetaCov/Wuhan/WH03/2020 & 36 - BetaCoV/Zhejiang/WZ-02/2020 & 603 \\
\hline $59-$ BetaCoV/France/IDF0373/2020 & $68-$ BetaCoV/Singapore $/ 2 / 2020$ & 602 \\
\hline $34-$ BetaCoV/Shenzhen/SZTH-004/2020 & $40-$ BetaCoV/Beijing/IV DC-BJ-005/2020 & 598 \\
\hline $36-$ BetaCoV/Zhejiang/WZ-02/2020 & $80-$ BetaCoV/Taiwan/NTU01/2020 & 597 \\
\hline $16-$ BetaCov/Wuhan/WH03/2020 & 33 - BetaCoV/Shenzhen/SZTH-003/2020 & 596 \\
\hline $39-$ BetaCoV/Chongqing/IVDC-CQ-00 1/2020 & $40-$ BetaCoV/Beijing/IV DC-BJ-005/2020 & 595 \\
\hline $16-$ BetaCov/Wuhan/WH03/2020 & $50-$ BetaCoV/Guangdong/20SF $174 / 2020$ & 595 \\
\hline $8-\mathrm{BetaCoV} /$ Wuhan/WIV06/2019 & $21-$ BetaCoV/Shenzhen/HKU-SZ-002/2020 & 594 \\
\hline $44-$ BetaCoV/Jiangsu/IVDC-JS-00 $1 / 2020$ & $46-\mathrm{BetaCoV} / \mathrm{USA} / \mathrm{IL} 1 / 2020$ & 592 \\
\hline $40-$ BetaCoV/Beijing/IV DC-BJ-005/2020 & $41-\mathrm{BetaCoV} / \mathrm{USA} / \mathrm{WA} 1 / 2020$ & 591 \\
\hline $79-$ BetaCoV/Australia/QLD02/2020 & $80-\mathrm{BetaCoV} /$ Taiwan/NTU01/2020 & 590 \\
\hline $64-\mathrm{BetaCoV} / J a p a n / A I / I-004 / 2020$ & 65 - BetaCoV/Korea/KCDC03/2020 & 588 \\
\hline $6-\mathrm{BetaCoV} /$ Wuhan/WIV02/2019 & $19-$ BetaCov/Wuhan/WH04/2020 & 587 \\
\hline $8-\mathrm{BetaCoV} /$ Wuhan/WIV06/2019 & 36 - BetaCoV/Zhejiang/WZ-02/2020 & 587 \\
\hline $9-$ BetaCoV/Wuhan/WIV07/2019 & $65-$ BetaCoV/Korea/KCDC03/2020 & 587 \\
\hline $42-$ BetaCoV/Hangzhou/HZCDC000 $1 / 2020$ & 79 - BetaCoV/Australia/QLD02/2020 & 587 \\
\hline $6-$ BetaCoV/Wuhan/WIV02/2019 & $42-$ BetaCoV/Hangzhou/HZCDCO00 1/2020 & 586 \\
\hline 2-BetaCov/Wuhan/WHO01/2019 & $32-$ BetaCoV/Zhejiang/WZ-0 1/2020 & 585 \\
\hline $19-$ BetaCov/Wuhan/WH04/2020 & 79 - BetaCoV/Australia/QLD02/2020 & 585 \\
\hline $8-\mathrm{BetaCoV} /$ Wuhan/WIV06/2019 & 33 - BetaCoV/Shenzhen/SZTH-003/2020 & 584 \\
\hline $55-$ BetaCoV/Taiwan $/ 2 / 2020$ & 58 - BetaCo V/France/IDF0372/2020 & 584 \\
\hline $7-\mathrm{BetaCoV} /$ Wuhan/WIV05/2019 & 62 - BetaCoV/Australia/NS WO $1 / 2020$ & 584 \\
\hline $34-$ BetaCoV/Shenzhen/SZTH-004/2020 & 50 - BetaCoV/Guangdong/20SF174/2020 & 583 \\
\hline $44-$ BetaCoV/Jiangsu/IVDC-JS-00 $1 / 2020$ & $59-\mathrm{BetaCoV} /$ France/IDF0373/2020 & 581 \\
\hline 76 - BetaCoV/USA/CA5/2020 & 77 - BetaCo V/France/IDF05 15/2020 & 580 \\
\hline $8-\mathrm{BetaCoV} /$ Wuhan/WIV06/2019 & $9-$ BetaCoV/Wuhan/WIV07/2019 & 579 \\
\hline $67-\mathrm{BetaCoV} / \mathrm{USA} / \mathrm{W}$ A $1-\mathrm{F} 6 / 2020$ & 68 - BetaCoV/Singapore/2/2020 & 579 \\
\hline 19 - BetaCov/Wuhan/WH04/2020 & 62 - BetaCoV/Australia/NS WO 1/2020 & 574 \\
\hline $15-\mathrm{BetaCoV} /$ Wuhan/IPBCAMS-WH-05/2020 & 17 - BetaCoV/China/WHUO1/2020 & 571 \\
\hline $57-$ BetaCoV/Guangdong/20SF 201/2020 & $81-$ BetaCoV/Singapore/3/2020 & 570 \\
\hline $19-$ BetaCov/Wuhan/WH04/2020 & 64 - BetaCoV/Japan/AI/I-004/2020 & 557 \\
\hline $68-\mathrm{BetaCoV} /$ Singapore $/ 2 / 2020$ & $69-$ BetaCoV/Germany/BavPat 1/2020 & 557 \\
\hline $70-$ BetaCoV/Australia/QLD0O1/2020 & 79 - BetaCoV/Australia/QLD02/2020 & 556 \\
\hline $44-$ BetaCoV/Jiangsu/IVDC-JS-00 $1 / 2020$ & $50-$ BetaCoV/Guangdong/20SF $174 / 2020$ & 551 \\
\hline $19-$ BetaCov/Wuhan/WH04/2020 & 33 - BetaCoV/Shenzhen/SZTH-003/2020 & 550 \\
\hline
\end{tabular}


Table 4. Edge weight between distinct nucleotide sequences in the Transcendental Information Cascade we constructed (contd.).

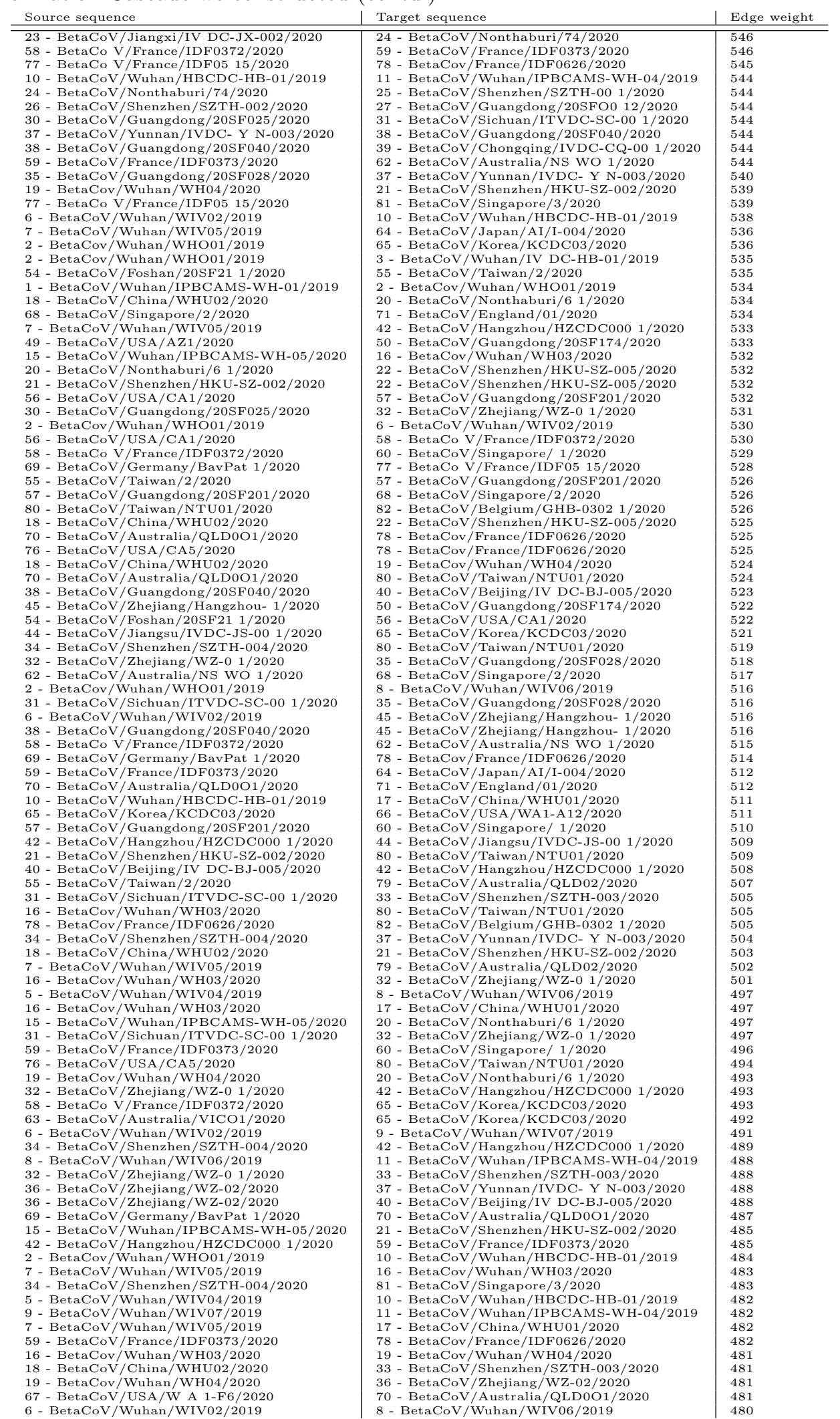


Table 5. Edge weight between distinct nucleotide sequences in the Transcendental Information Cascade we constructed (contd.).

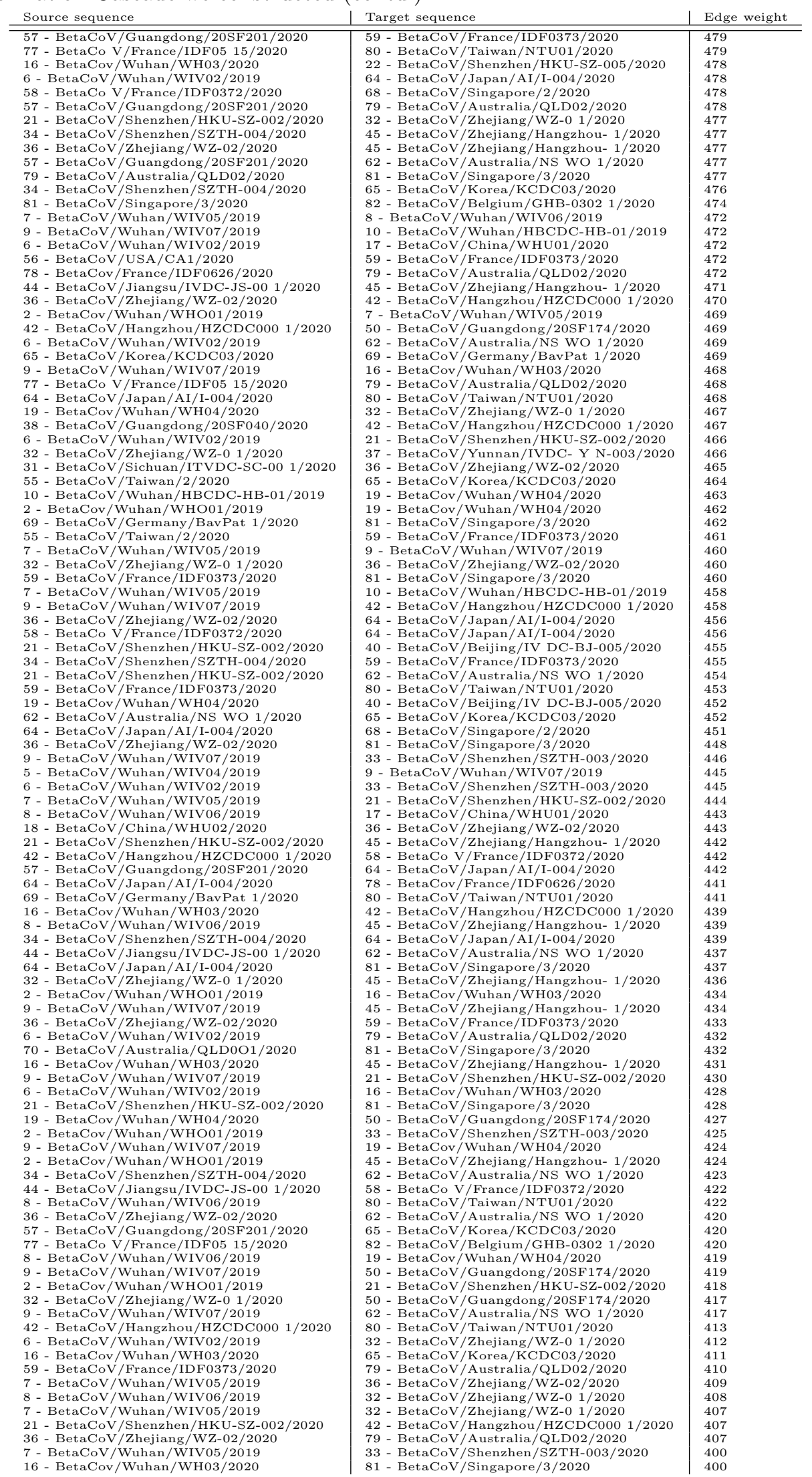


Table 6. Edge weight between distinct nucleotide sequences in the Transcendental Information Cascade we constructed (contd.).

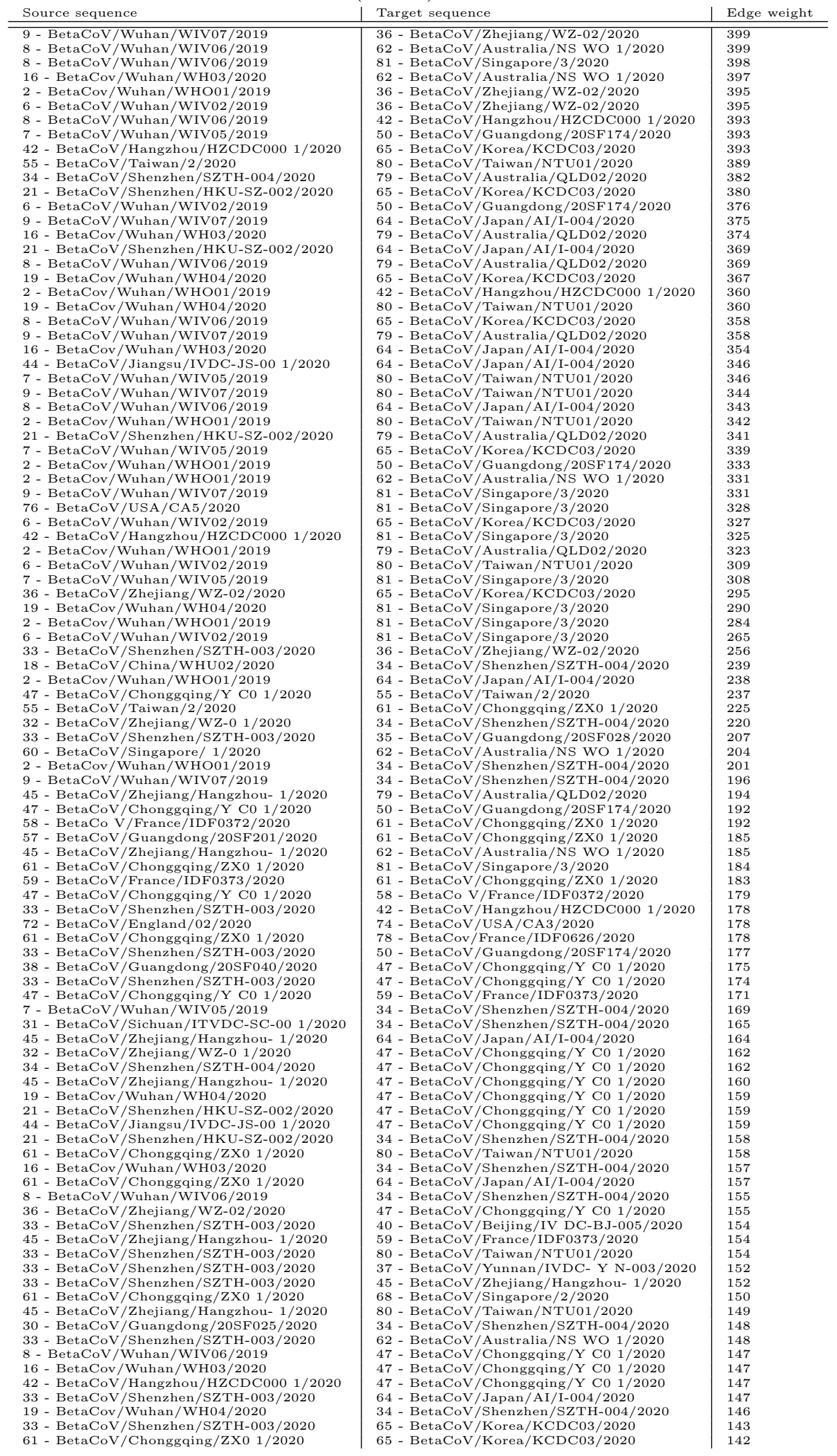


medRxiv preprint doi: https://doi.org/10.1101/2020.02.07.20021139; this version posted February 17, 2020. The copyright holder for this preprint (which was not certified by peer review) is the author/funder, who has granted medRxiv a license to display the preprint in perpetuity.

It is made available under a CC-BY 4.0 International license .

Table 7. Edge weight between distinct nucleotide sequences in the Transcendental Information Cascade we constructed (contd.).

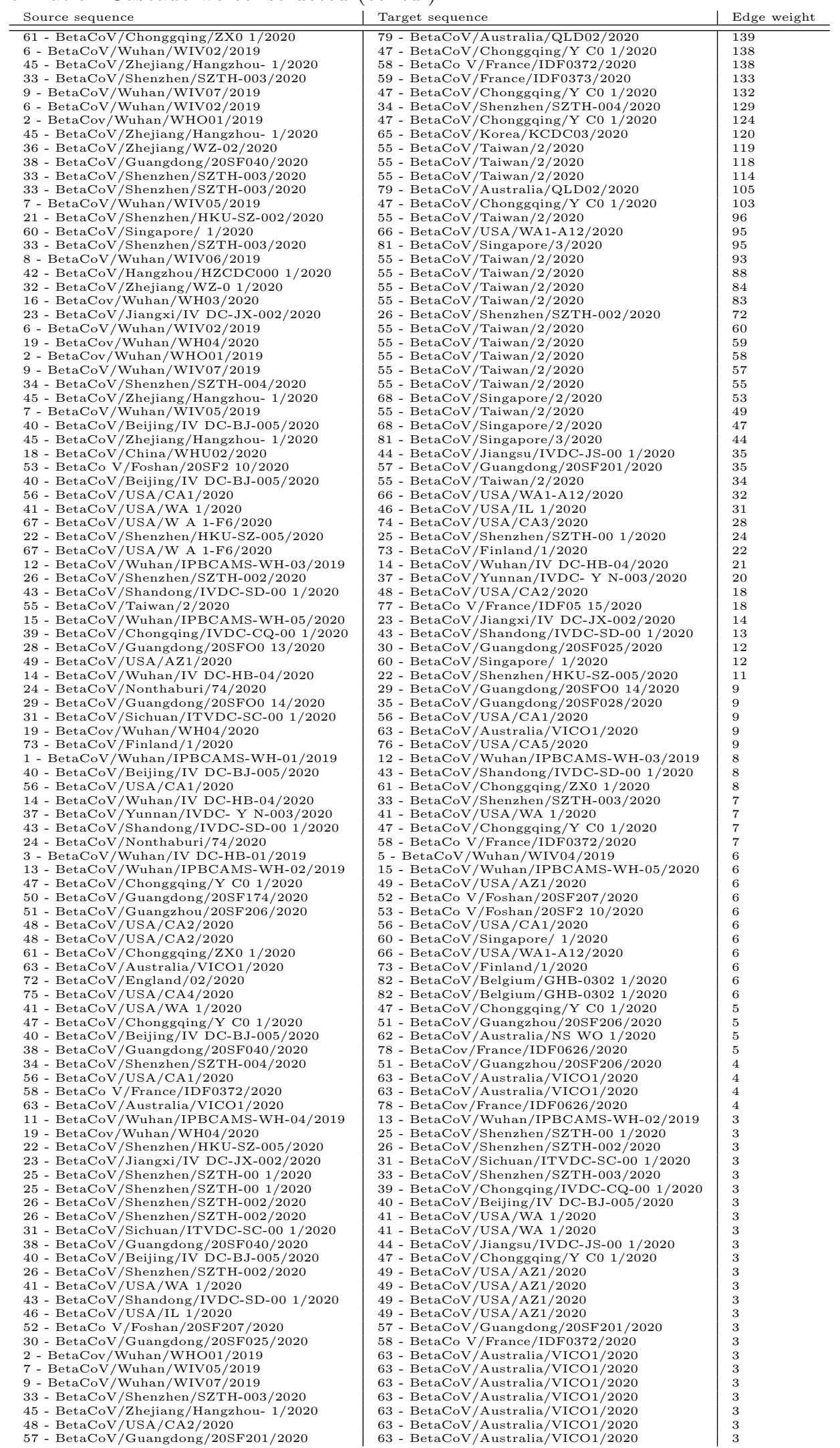


Table 8. Edge weight between distinct nucleotide sequences in the Transcendental Information Cascade we constructed (contd.).

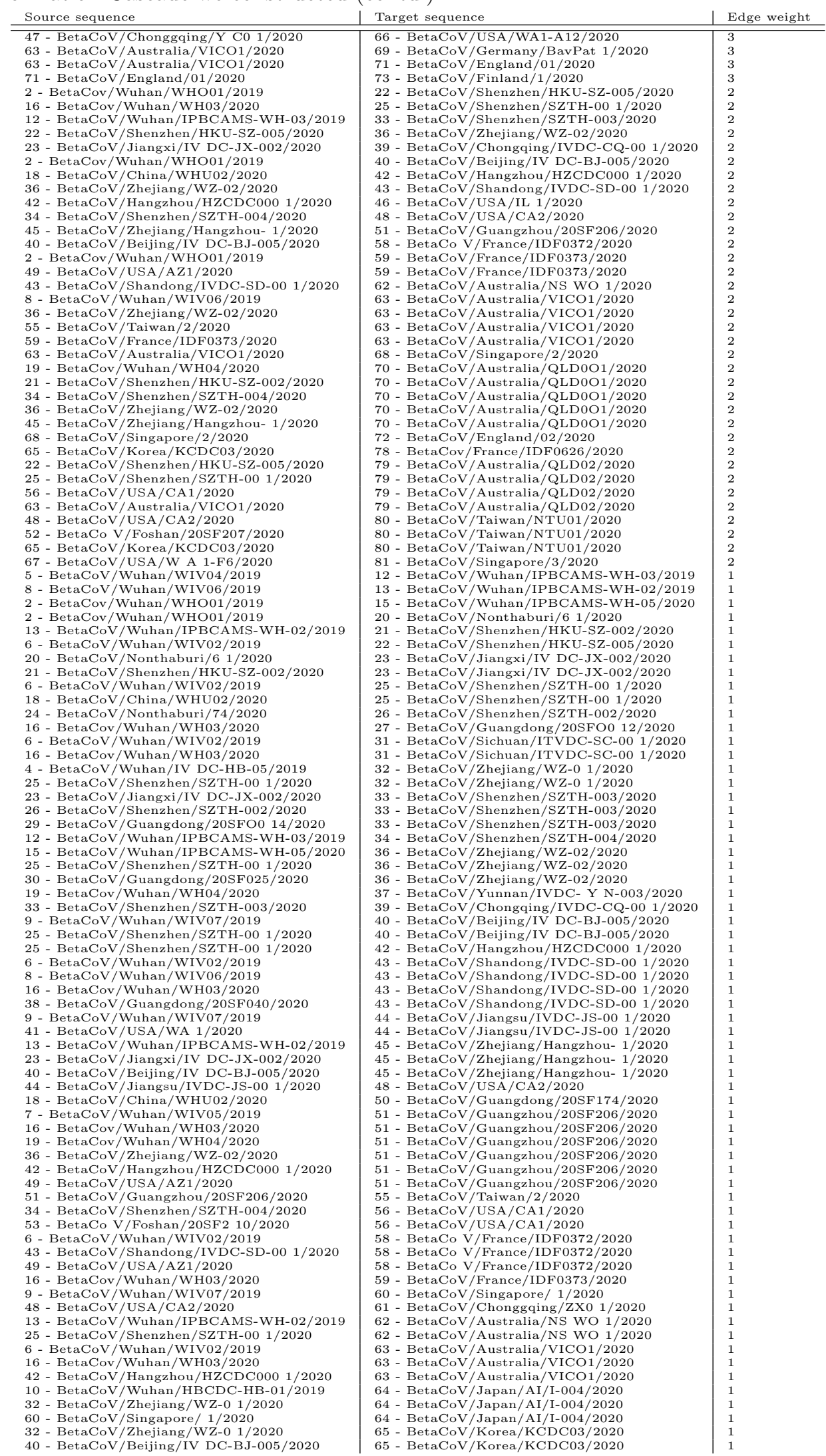


Table 9. Edge weight between distinct nucleotide sequences in the Transcendental Information Cascade we constructed (contd.).

\begin{tabular}{|c|c|c|}
\hline Source sequence & Target sequence & Edge weight \\
\hline $60-$ BetaCoV/Singapore/ 1/2020 & $65-$ BetaCoV/Korea/KCDC03/2020 & 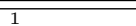 \\
\hline 58 - BetaCo V/France/IDF0372/2020 & $66-\mathrm{BetaCoV} / \mathrm{USA} / \mathrm{WA} 1-\mathrm{A} 12 / 2020$ & 1 \\
\hline 62 - BetaCoV/Australia/NS WO $1 / 2020$ & $66-\mathrm{BetaCoV} / \mathrm{USA} / \mathrm{WA} 1-\mathrm{A} 12 / 2020$ & 1 \\
\hline $18-$ BetaCoV/China/WHU02/2020 & $68-$ BetaCoV/Singapore/2/2020 & 1 \\
\hline 36 - BetaCoV/Zhejiang/WZ-02/2020 & $68-$ BetaCoV/Singapore/2/2020 & 1 \\
\hline 42 - BetaCoV/Hangzhou/HZCDC000 1/2020 & $68-$ BetaCoV/Singapore/2/2020 & 1 \\
\hline $2-$ BetaCov/Wuhan/WHO01/2019 & $69-$ BetaCoV/Germany/BavPat 1/2020 & 1 \\
\hline $16-$ BetaCov/Wuhan/WH03/2020 & $69-$ BetaCoV/Germany/BavPat $1 / 2020$ & 1 \\
\hline $19-$ BetaCov/Wuhan/WH04/2020 & $69-$ BetaCoV/Germany/BavPat $1 / 2020$ & 1 \\
\hline 57 - BetaCoV/Guangdong/20SF201/2020 & $69-$ BetaCoV/Germany/BavPat $1 / 2020$ & 1 \\
\hline 2 - BetaCov/Wuhan/WHO01/2019 & 70 - BetaCoV/Australia/QLD0O1/2020 & 1 \\
\hline 7 - BetaCoV/Wuhan/WIV05/2019 & $70-$ BetaCoV/Australia/QLD0O1/2020 & 1 \\
\hline 8 - BetaCoV/Wuhan/WIV06/2019 & $70-$ BetaCoV/Australia/QLD0O1/2020 & 1 \\
\hline 33 - BetaCoV/Shenzhen/SZTH-003/2020 & 70 - BetaCoV/Australia/QLD0O1/2020 & 1 \\
\hline 58 - BetaCo V/France/IDF0372/2020 & 70 - BetaCoV/Australia/QLD0O1/2020 & 1 \\
\hline $61-$ BetaCoV/Chonggqing/ZX0 $1 / 2020$ & 70 - BetaCoV/Australia/QLD0O1/2020 & 1 \\
\hline $64-\mathrm{BetaCoV} / \mathrm{Japan} / \mathrm{AI} / \mathrm{I}-004 / 2020$ & 70 - BetaCoV/Australia/QLD0O1/2020 & 1 \\
\hline $34-$ BetaCoV/Shenzhen/SZTH-004/2020 & $71-$ BetaCoV/England/01/2020 & 1 \\
\hline $45-$ BetaCoV/Zhejiang/Hangzhou- $1 / 2020$ & $71-$ BetaCoV/England/01/2020 & 1 \\
\hline $55-$ BetaCoV/Taiwan/2/2020 & $71-$ BetaCoV/England/01/2020 & 1 \\
\hline $19-$ BetaCov/Wuhan/WH04/2020 & $72-$ BetaCoV/England/02/2020 & 1 \\
\hline 21 - BetaCoV/Shenzhen/HKU-SZ-002/2020 & $72-$ BetaCoV/England/02/2020 & 1 \\
\hline $67-\mathrm{BetaCoV} / \mathrm{USA} / \mathrm{W}$ A $1-\mathrm{F} 6 / 2020$ & $72-$ BetaCoV/England/02/2020 & 1 \\
\hline $2-$ BetaCov/Wuhan/WHO01/2019 & $74-\mathrm{BetaCoV} / \mathrm{USA} / \mathrm{CA} 3 / 2020$ & 1 \\
\hline $57-$ BetaCoV/Guangdong/20SF201/2020 & $76-\mathrm{BetaCoV} / \mathrm{USA} / \mathrm{CA} 5 / 2020$ & 1 \\
\hline 19 - BetaCov/Wuhan/WH04/2020 & 77 - BetaCo V/France/IDF05 15/2020 & 1 \\
\hline 58 - BetaCo V/France/IDF0372/2020 & 77 - BetaCo V/France/IDF05 $15 / 2020$ & 1 \\
\hline $59-$ BetaCoV/France/IDF0373/2020 & 77 - BetaCo V/France/IDF05 $15 / 2020$ & 1 \\
\hline $61-$ BetaCoV/Chonggqing/ZXo $1 / 2020$ & 77 - BetaCo V/France/IDF05 15/2020 & 1 \\
\hline $55-$ BetaCoV/Taiwan/2/2020 & 78 - BetaCov/France/IDF0626/2020 & 1 \\
\hline 14 - BetaCoV/Wuhan/IV DC-HB-04/2020 & 79 - BetaCoV/Australia/QLD02/2020 & 1 \\
\hline $31-$ BetaCoV/Sichuan/ITVDC-SC-00 1/2020 & 79 - BetaCoV/Australia/QLD02/2020 & 1 \\
\hline 32 - BetaCoV/Zhejiang/WZ-0 1/2020 & 79 - BetaCoV/Australia/QLD02/2020 & 1 \\
\hline 13 - BetaCoV/Wuhan/IPBCAMS-WH-02/2019 & $80-\mathrm{BetaCoV} /$ Taiwan/NTU01/2020 & 1 \\
\hline 60 - BetaCoV/Singapore/ 1/2020 & $80-$ BetaCoV/Taiwan/NTU01/2020 & 1 \\
\hline
\end{tabular}


medRxiv preprint doi: https://doi.org/10.1101/2020.02.07.20021139; this version posted February 17, 2020. The copyright holder for this preprint (which was not certified by peer review) is the author/funder, who has granted medRxiv a license to display the preprint in perpetuity.

It is made available under a CC-BY 4.0 International license.

Table 10. Frequency of edge distances.

\begin{tabular}{|c|c|}
\hline Edge distance & Frequency \\
\hline 1 & 801289 \\
\hline 2 & 197353 \\
\hline 3 & 166842 \\
\hline 4 & 123821 \\
\hline 5 & 92366 \\
\hline 6 & 88539 \\
\hline 7 & 87805 \\
\hline 20 & 55715 \\
\hline 10 & 51884 \\
\hline 9 & 32851 \\
\hline 22 & 27681 \\
\hline 27 & 26443 \\
\hline 24 & 24707 \\
\hline 14 & 11886 \\
\hline 26 & 7172 \\
\hline 11 & 7013 \\
\hline 13 & 6058 \\
\hline 8 & 5205 \\
\hline 15 & 3772 \\
\hline 12 & 3304 \\
\hline 17 & 3124 \\
\hline 23 & 2893 \\
\hline 16 & 2240 \\
\hline 43 & 2168 \\
\hline 25 & 2044 \\
\hline 29 & 1968 \\
\hline 21 & 1892 \\
\hline 19 & 1811 \\
\hline 28 & 1807 \\
\hline 34 & 1682 \\
\hline 31 & 1623 \\
\hline 18 & 1601 \\
\hline 45 & 1511 \\
\hline 46 & 1445 \\
\hline 30 & 1422 \\
\hline 56 & 1402 \\
\hline 44 & 1363 \\
\hline 60 & 1344 \\
\hline 72 & 1256 \\
\hline 73 & 1176 \\
\hline 38 & 1172 \\
\hline 58 & 1159 \\
\hline 36 & 1125 \\
\hline 39 & 1071 \\
\hline 37 & 1030 \\
\hline 41 & 1011 \\
\hline 55 & 961 \\
\hline 63 & 911 \\
\hline 57 & 899 \\
\hline 59 & 836 \\
\hline 48 & 832 \\
\hline 47 & 732 \\
\hline 71 & 713 \\
\hline 35 & 685 \\
\hline 74 & 617 \\
\hline 42 & 616 \\
\hline 62 & 529 \\
\hline 64 & 505 \\
\hline 53 & 477 \\
\hline 49 & 474 \\
\hline 40 & 468 \\
\hline 33 & 459 \\
\hline 54 & 405 \\
\hline 65 & 401 \\
\hline 61 & 363 \\
\hline 70 & 358 \\
\hline 32 & 351 \\
\hline 78 & 342 \\
\hline 77 & 323 \\
\hline 79 & 284 \\
\hline 75 & 265 \\
\hline 51 & 4 \\
\hline 50 & 2 \\
\hline 67 & 2 \\
\hline 52 & 1 \\
\hline 68 & 1 \\
\hline
\end{tabular}


medRxiv preprint doi: https://doi.org/10.1101/2020.02.07.20021139; this version posted February 17, 2020. The copyright holder for this preprint (which was not certified by peer review) is the author/funder, who has granted medRxiv a license to display the preprint in perpetuity. It is made available under a CC-BY 4.0 International license .

Table 11. Cluster memberships of the nucleotide sequences (full TIC network).

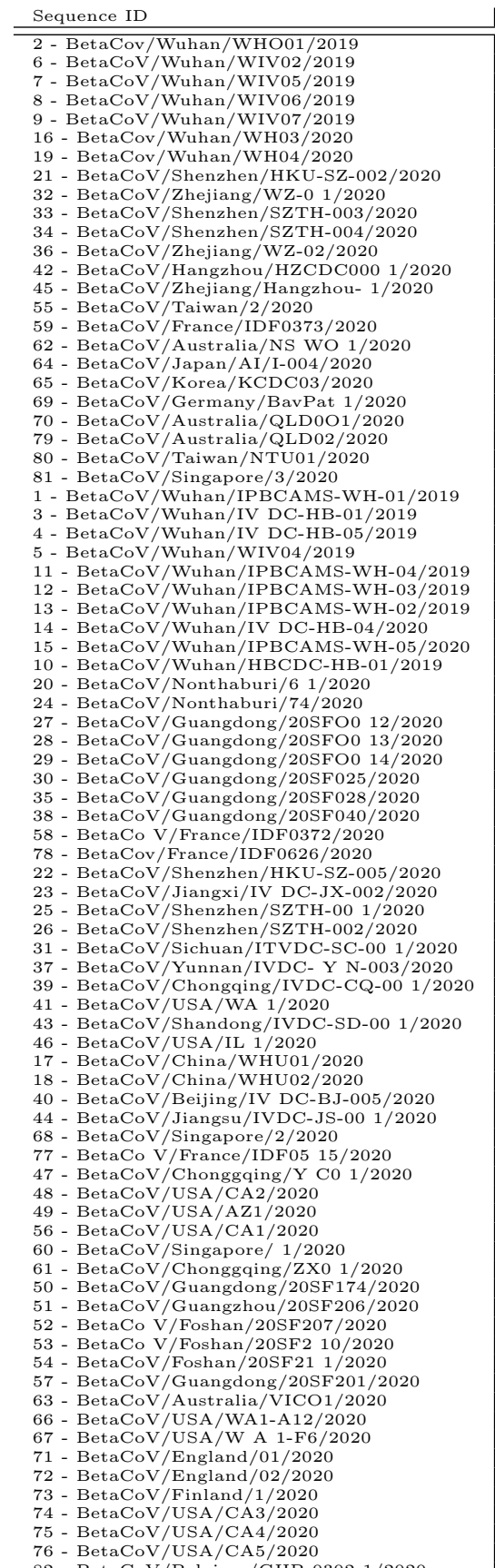

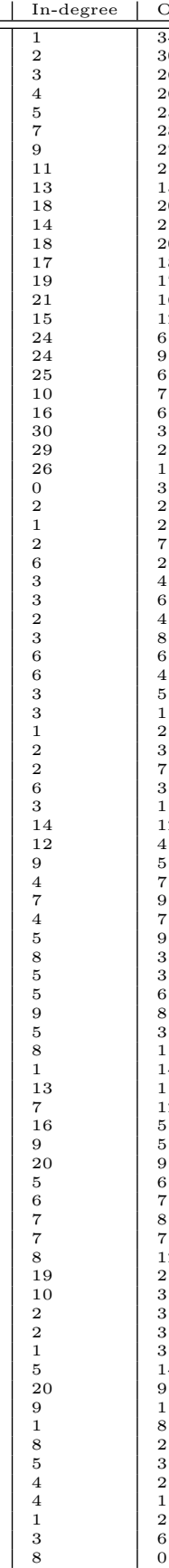

\begin{tabular}{|c|c|}
\hline 34 & 35 \\
\hline 30 & 32 \\
\hline 26 & 29 \\
\hline 26 & 30 \\
\hline 25 & 30 \\
\hline 28 & 35 \\
\hline 27 & 36 \\
\hline 21 & 32 \\
\hline 15 & 28 \\
\hline 20 & 38 \\
\hline 21 & 35 \\
\hline 20 & 38 \\
\hline 18 & 35 \\
\hline 17 & 36 \\
\hline 16 & 37 \\
\hline 12 & 27 \\
\hline 6 & 30 \\
\hline 9 & 33 \\
\hline 6 & 31 \\
\hline 7 & 17 \\
\hline 6 & 22 \\
\hline 3 & 33 \\
\hline 2 & 31 \\
\hline 1 & 27 \\
\hline 3 & 3 \\
\hline 2 & 4 \\
\hline 2 & 3 \\
\hline 7 & 9 \\
\hline 2 & 8 \\
\hline 4 & 7 \\
\hline 6 & 9 \\
\hline 4 & 6 \\
\hline 8 & 11 \\
\hline 6 & 12 \\
\hline 4 & 10 \\
\hline 5 & 8 \\
\hline 1 & 4 \\
\hline 2 & 3 \\
\hline 3 & 5 \\
\hline 7 & 9 \\
\hline 3 & 9 \\
\hline 11 & 14 \\
\hline 12 & 26 \\
\hline 4 & 16 \\
\hline 5 & 14 \\
\hline 7 & 11 \\
\hline 9 & 16 \\
\hline 7 & 11 \\
\hline 9 & 14 \\
\hline 3 & 11 \\
\hline 3 & 8 \\
\hline 6 & 11 \\
\hline 8 & 17 \\
\hline 3 & 8 \\
\hline 1 & 9 \\
\hline 14 & 15 \\
\hline 11 & 24 \\
\hline 12 & 19 \\
\hline 5 & 21 \\
\hline 5 & 14 \\
\hline 9 & 29 \\
\hline 6 & 11 \\
\hline 7 & 13 \\
\hline 8 & 15 \\
\hline 7 & 14 \\
\hline 12 & 20 \\
\hline 2 & 21 \\
\hline 3 & 13 \\
\hline 3 & 5 \\
\hline 3 & 5 \\
\hline 3 & 4 \\
\hline 14 & 19 \\
\hline 9 & 29 \\
\hline 1 & 10 \\
\hline 8 & 9 \\
\hline 2 & 10 \\
\hline 3 & 8 \\
\hline 2 & 6 \\
\hline 1 & 5 \\
\hline 2 & 3 \\
\hline 6 & 9 \\
\hline 0 & 8 \\
\hline
\end{tabular}


medRxiv preprint doi: https://doi.org/10.1101/2020.02.07.20021139; this version posted February 17, 2020. The copyright holder for this preprint (which was not certified by peer review) is the author/funder, who has granted medRxiv a license to display the preprint in perpetuity. It is made available under a CC-BY 4.0 International license.

Table 12. Inter-cluster similarities for the full TIC network.

\begin{tabular}{c|c|c|c|c} 
Inter-cluster pair & Avg. similarity & SD & Min. similarity & Max. similarity \\
\hline \hline $0-1$ & 0.267773 & 0.013962 & 0.252325 & 0.293298 \\
$0-2$ & 0.265648 & 0.012247 & 0.251072 & 0.288222 \\
$0-3$ & 0.267719 & 0.013965 & 0.252326 & 0.293298 \\
$0-4$ & 0.267501 & 0.021119 & 0.250904 & 0.358635 \\
$0-5$ & 0.267639 & 0.013011 & 0.252337 & 0.293264 \\
$0-6$ & 0.265093 & 0.013211 & 0.251181 & 0.288327 \\
$0-7$ & 0.267556 & 0.013985 & 0.250745 & 0.293466 \\
$1-2$ & 0.259499 & 0.000047 & 0.259407 & 0.259605 \\
$1-3$ & 0.999513 & 0.000290 & 0.998796 & 0.999900 \\
$1-4$ & 0.269435 & 0.000147 & 0.269120 & 0.269655 \\
$1-5$ & 0.927233 & 0.103628 & 0.781755 & 0.999966 \\
$1-6$ & 0.258528 & 0.000033 & 0.258454 & 0.258598 \\
$1-7$ & 0.998823 & 0.001846 & 0.993938 & 0.999933 \\
$2-3$ & 0.259472 & 0.000053 & 0.259373 & 0.259572 \\
$2-4$ & 0.255754 & 0.000079 & 0.255587 & 0.255887 \\
$2-5$ & 0.260785 & 0.001855 & 0.259407 & 0.263405 \\
$2-6$ & 0.284333 & 0.000043 & 0.284259 & 0.284454 \\
$2-7$ & 0.259330 & 0.000421 & 0.258067 & 0.259682 \\
$3-4$ & 0.269369 & 0.000184 & 0.268965 & 0.269823 \\
$3-5$ & 0.926970 & 0.103499 & 0.781540 & 0.999967 \\
$3-6$ & 0.258482 & 0.000049 & 0.258387 & 0.258598 \\
$3-7$ & 0.998632 & 0.001816 & 0.993235 & 1.000000 \\
$4-5$ & 0.268894 & 0.000682 & 0.267715 & 0.269603 \\
$4-6$ & 0.263907 & 0.000187 & 0.263414 & 0.264057 \\
$4-7$ & 0.269143 & 0.000600 & 0.267290 & 0.269928 \\
$5-6$ & 0.260599 & 0.002943 & 0.258521 & 0.264821 \\
$5-7$ & 0.926460 & 0.103206 & 0.779396 & 0.999900 \\
$6-7$ & 0.258383 & 0.000525 & 0.256844 & 0.258889
\end{tabular}

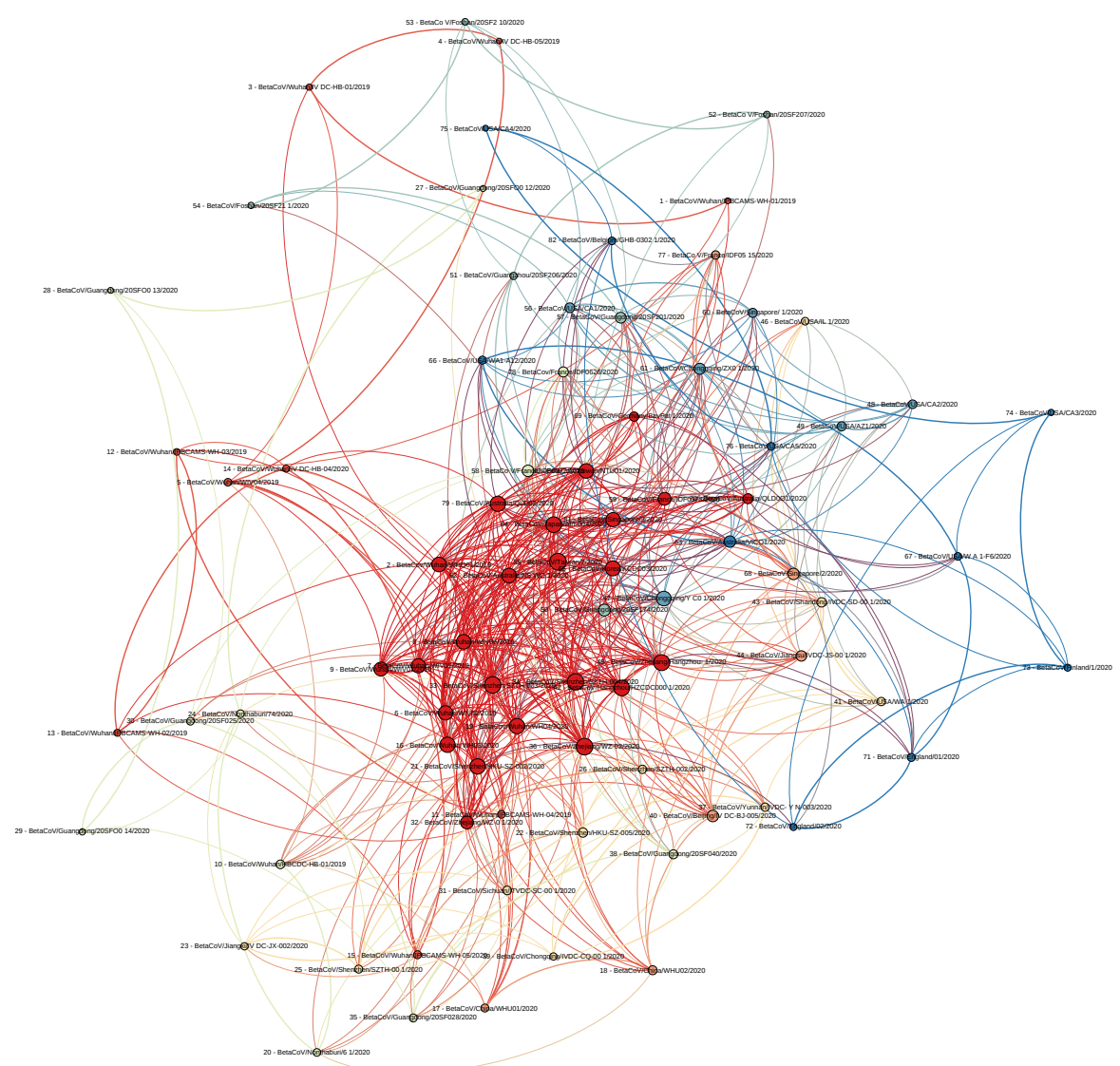

Figure 6. +1 reading frame network. 
medRxiv preprint doi: https://doi.org/10.1101/2020.02.07.20021139; this version posted February 17, 2020. The copyright holder for this preprint (which was not certified by peer review) is the author/funder, who has granted medRxiv a license to display the preprint in perpetuity. It is made available under a CC-BY 4.0 International license .

Table 13. Cluster memberships of the nucleotide sequences (network for +1 reading frames).

\begin{tabular}{|c|c|c|c|c|}
\hline Sequence ID & In-degree & Out-degree & Degree & Cluster \\
\hline 2 - BetaCov/Wuhan/WHO01/2019 & 1 & 27 & 28 & 0 \\
\hline 6- BetaCoV/Wuhan/WIV02/2019 & 2 & 25 & 27 & 0 \\
\hline 7 - BetaCoV/Wuhan/WIV05/2019 & 3 & 24 & 27 & 0 \\
\hline 8 - BetaCoV/Wuhan/WIV06/2019 & 4 & 24 & 28 & 0 \\
\hline 9 - BetaCoV/Wuhan/WIV07/2019 & 5 & 21 & 26 & 0 \\
\hline 16 - BetaCov/Wuhan/WH03/2020 & 7 & 23 & 30 & 0 \\
\hline 19 - BetaCov/Wuhan/WH04/2020 & 9 & 21 & 30 & 0 \\
\hline $21-$ BetaCoV/Shenzhen/HKU-SZ-002/2020 & 11 & 19 & 30 & 0 \\
\hline 32 - BetaCoV/Zhejiang/WZ-0 1/2020 & 11 & 12 & 23 & 0 \\
\hline $33-\mathrm{BetaCoV} /$ Shenzhen/SZTH-003/2020 & 15 & 19 & 34 & 0 \\
\hline $34-$ BetaCoV/Shenzhen/SZTH-004/2020 & 13 & 20 & 33 & 0 \\
\hline $36-$ BetaCoV/Zhejiang/WZ-02/2020 & 16 & 18 & 34 & 0 \\
\hline 42 - BetaCoV/Hangzhou/HZCDC000 1/2020 & 16 & 15 & 31 & 0 \\
\hline $45-$ BetaCoV/Zhejiang/Hangzhou- $1 / 2020$ & 17 & 15 & 32 & 0 \\
\hline $55-\mathrm{BetaCoV} /$ Taiwan $/ 2 / 2020$ & 20 & 14 & 34 & 0 \\
\hline $59-$ BetaCoV/France/IDF0373/2020 & 13 & 10 & 23 & 0 \\
\hline $62-$ BetaCoV/Australia/NS WO 1/2020 & 22 & 6 & 28 & 0 \\
\hline $64-$ BetaCoV/Japan/AI/I-004/2020 & 22 & 9 & 31 & 0 \\
\hline $65-\mathrm{BetaCoV} /$ Korea/KCDC03/2020 & 24 & 5 & 29 & 0 \\
\hline $69-$ BetaCoV/Germany/BavPat $1 / 2020$ & 6 & 7 & 13 & 0 \\
\hline 70 - BetaCoV/Australia/QLD0O1/2020 & 9 & 6 & 15 & 0 \\
\hline 79 - BetaCoV/Australia/QLD02/2020 & 27 & 3 & 30 & 0 \\
\hline $80-$ BetaCoV/Taiwan/NTU01/2020 & 26 & 2 & 28 & 0 \\
\hline $81-$ BetaCoV/Singapore/3/2020 & 26 & 1 & 27 & 0 \\
\hline 1 - BetaCoV/Wuhan/IPBCAMS-WH-01/2019 & 0 & 3 & 3 & 1 \\
\hline $3-\mathrm{BetaCoV} /$ Wuhan/IV DC-HB-01/2019 & 2 & 2 & 4 & 1 \\
\hline 4 - BetaCoV/Wuhan/IV DC-HB-05/2019 & 1 & 1 & 2 & 1 \\
\hline 5 - BetaCoV/Wuhan/WIV04/2019 & 2 & 6 & 8 & 1 \\
\hline $11-\mathrm{BetaCoV} /$ Wuhan/IPBCAMS-WH-04/2019 & 6 & 2 & 8 & 1 \\
\hline $12-\mathrm{BetaCoV} / \mathrm{Wuhan} / \mathrm{IPBCAMS}-\mathrm{WH}-03 / 2019$ & 2 & 3 & 5 & 1 \\
\hline $13-\mathrm{BetaCoV} / \mathrm{Wuhan} / \mathrm{IPBCAMS}-\mathrm{WH}-02 / 2019$ & 2 & 4 & 6 & 1 \\
\hline $14-\mathrm{BetaCoV} / \mathrm{Wuhan} / \mathrm{IV}$ DC-HB-04/2020 & 2 & 4 & 6 & 1 \\
\hline $15-\mathrm{BetaCoV} / \mathrm{Wuhan} / \mathrm{IPBCAMS}-\mathrm{WH}-05 / 2020$ & 2 & 7 & 9 & 1 \\
\hline $17-\mathrm{BetaCoV} /$ China/WHU01/2020 & 8 & 1 & 9 & 2 \\
\hline 18 - BetaCoV/China/WHU02/2020 & 1 & 11 & 12 & 2 \\
\hline $40-$ BetaCoV/Beijing/IV DC-BJ-005/2020 & 10 & 9 & 19 & 2 \\
\hline $44-\mathrm{BetaCoV} / J i a n g s u / I V D C-J S-001 / 2020$ & 4 & 12 & 16 & 2 \\
\hline $68-$ BetaCoV/Singapore/2/2020 & 14 & 5 & 19 & 2 \\
\hline 77 - BetaCo V/France/IDF05 15/2020 & 5 & 5 & 10 & 2 \\
\hline $22-$ BetaCoV/Shenzhen/HKU-SZ-005/2020 & 8 & 5 & 13 & 3 \\
\hline $23-$ BetaCoV/Jiangxi/IV DC-JX-002/2020 & 2 & 5 & 7 & 3 \\
\hline $25-$ BetaCoV/Shenzhen/SZTH-00 1/2020 & 4 & 5 & 9 & 3 \\
\hline $26-$ BetaCoV/Shenzhen/SZTH-002/2020 & 4 & 6 & 10 & 3 \\
\hline $31-$ BetaCoV/Sichuan/ITVDC-SC-00 1/2020 & 3 & 8 & 11 & 3 \\
\hline $37-$ BetaCoV/Yunnan/IVDC- Y N-003/2020 & 7 & 3 & 10 & 3 \\
\hline 39 - BetaCoV/Chongqing/IVDC-CQ-00 1/2020 & 4 & 3 & 7 & 3 \\
\hline $41-\mathrm{BetaCoV} / \mathrm{USA} / \mathrm{WA} 1 / 2020$ & 5 & 5 & 10 & 3 \\
\hline $43-$ BetaCoV/Shandong/IVDC-SD-00 1/2020 & 7 & 6 & 13 & 3 \\
\hline $46-\mathrm{BetaCoV} / \mathrm{USA} / \mathrm{IL} 1 / 2020$ & 5 & 3 & 8 & 3 \\
\hline $10-$ BetaCoV/Wuhan/HBCDC-HB-01/2019 & 6 & 5 & 11 & 4 \\
\hline 20 - BetaCoV/Nonthaburi/6 1/2020 & 5 & 3 & 8 & 4 \\
\hline $24-$ BetaCoV/Nonthaburi/74/2020 & 3 & 5 & 8 & 4 \\
\hline 27 - BetaCoV/Guangdong/20SFOO 12/2020 & 2 & 1 & 3 & 4 \\
\hline 28 - BetaCoV/Guangdong/2OSFOO $13 / 2020$ & 1 & 2 & 3 & 4 \\
\hline 29 - BetaCoV/Guangdong/20SFOO 14/2020 & 2 & 2 & 4 & 4 \\
\hline $30-$ BetaCoV/Guangdong/20SF $025 / 2020$ & 2 & 6 & 8 & 4 \\
\hline 35 - BetaCoV/Guangdong/20SF028/2020 & 6 & 3 & 9 & 4 \\
\hline 38 - BetaCoV/Guangdong/20SF040/2020 & 3 & 9 & 12 & 4 \\
\hline 58 - BetaCo V/France/IDF0372/2020 & 11 & 8 & 19 & 4 \\
\hline $78-$ BetaCov/France/IDF0626/2020 & 11 & 4 & 15 & 4 \\
\hline 50 - BetaCoV/Guangdong/20SF 174/2020 & 18 & 2 & 20 & 5 \\
\hline 51 - BetaCoV/Guangzhou/20SF 206/2020 & 5 & 2 & 7 & 5 \\
\hline 52 - BetaCo V/Foshan/20SF 207/2020 & 2 & 3 & 5 & 5 \\
\hline 53 - BetaCo V/Foshan/20SF2 10/2020 & 2 & 3 & 5 & 5 \\
\hline $54-$ BetaCoV/Foshan/20SF21 1/2020 & 1 & 3 & 4 & 5 \\
\hline 57 - BetaCoV/Guangdong/20SF201/2020 & 5 & 12 & 17 & 5 \\
\hline $47-$ BetaCoV/Chonggqing/Y CO $1 / 2020$ & 19 & 9 & 28 & 6 \\
\hline $48-\mathrm{BetaCoV} / \mathrm{USA} / \mathrm{CA} 2 / 2020$ & 5 & 6 & 11 & 6 \\
\hline $49-\mathrm{BetaCoV} / \mathrm{USA} / \mathrm{AZ} 1 / 2020$ & 6 & 6 & 12 & 6 \\
\hline $56-\mathrm{BetaCoV} / \mathrm{USA} / \mathrm{CA} 1 / 2020$ & 6 & 8 & 14 & 6 \\
\hline $60-$ BetaCoV/Singapore/ $1 / 2020$ & 6 & 6 & 12 & 6 \\
\hline $61-$ BetaCoV/Chonggqing/ZX0 $1 / 2020$ & 8 & 10 & 18 & 6 \\
\hline $63-\mathrm{BetaCoV} /$ Australia/VICO1/2020 & 12 & 7 & 19 & 7 \\
\hline $66-\mathrm{BetaCoV} / \mathrm{USA} / \mathrm{WA} 1-\mathrm{A} 12 / 2020$ & 8 & 1 & 9 & 7 \\
\hline $67-\mathrm{BetaCoV} / \mathrm{USA} / \mathrm{W}$ A $1-\mathrm{F} 6 / 2020$ & 1 & 7 & 8 & 7 \\
\hline $71-$ BetaCoV/England/01/2020 & 7 & 2 & 9 & 7 \\
\hline $72-$ BetaCoV/England $/ 02 / 2020$ & 3 & 3 & 6 & 7 \\
\hline $73-$ BetaCoV/Finland/1/2020 & 4 & 2 & 6 & 7 \\
\hline $74-\mathrm{BetaCoV} / \mathrm{USA} / \mathrm{CA} 3 / 2020$ & 3 & 1 & 4 & 7 \\
\hline $75-\mathrm{BetaCoV} / \mathrm{USA} / \mathrm{CA} 4 / 2020$ & 1 & 2 & 3 & 7 \\
\hline $76-\mathrm{BetaCoV} / \mathrm{USA} / \mathrm{CA} 5 / 2020$ & 2 & 6 & 8 & 7 \\
\hline $82-$ BetaCoV/Belgium/GHB-0302 1/2020 & 8 & 0 & 8 & 7 \\
\hline
\end{tabular}

Table 14. Intra-cluster similarities (network for +1 reading frames).

\begin{tabular}{c|c|c|c|c|c} 
Cluster & Avg. similarity & SD & Min. similarity & Max. similarity & Cluster size \\
\hline \hline 0 & 0.277353 & 0.088247 & 0.250620 & 0.999866 & 24 \\
1 & 0.999730 & 0.000222 & 0.999231 & 1.000000 & 9 \\
2 & 0.998768 & 0.001345 & 0.996387 & 1.000000 & 6 \\
3 & 0.999385 & 0.000371 & 0.998461 & 0.999933 & 10 \\
4 & 0.999915 & 0.000048 & 0.999832 & 1.000000 & 11 \\
5 & 0.999926 & 0.000036 & 0.999866 & 1.000000 & 6 \\
6 & 0.883701 & 0.112398 & 0.781976 & 0.999899 & 6 \\
7 & 0.997900 & 0.002419 & 0.991158 & 1.000000 & 10
\end{tabular}


medRxiv preprint doi: https://doi.org/10.1101/2020.02.07.20021139; this version posted February 17, 2020. The copyright holder for this preprint (which was not certified by peer review) is the author/funder, who has granted medRxiv a license to display the preprint in perpetuity. It is made available under a CC-BY 4.0 International license .

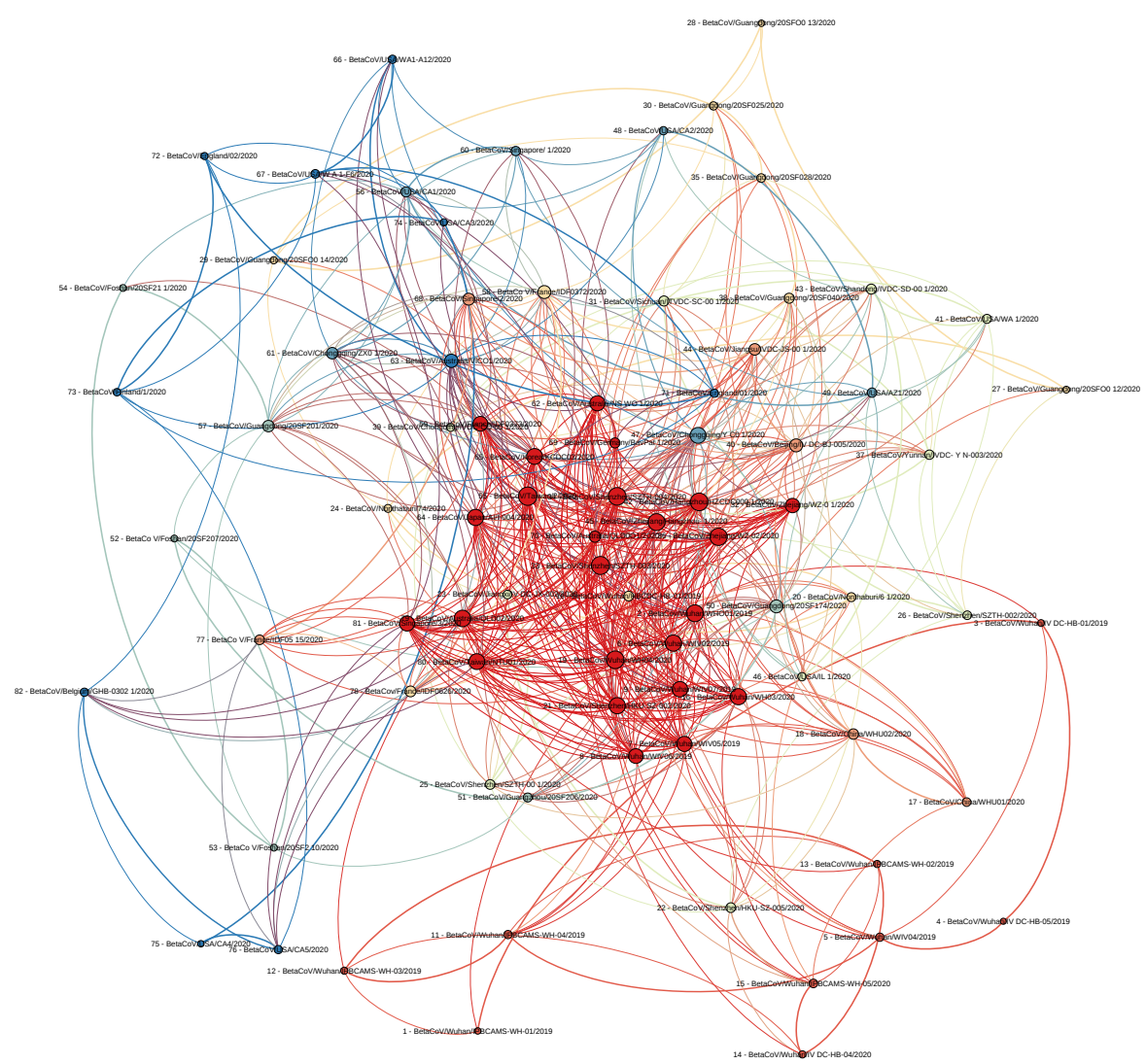

Figure 7. +2 reading frame network. 
medRxiv preprint doi: https://doi.org/10.1101/2020.02.07.20021139; this version posted February 17, 2020. The copyright holder for this preprint (which was not certified by peer review) is the author/funder, who has granted medRxiv a license to display the preprint in perpetuity. It is made available under a CC-BY 4.0 International license .

Table 15. Cluster memberships of the nucleotide sequences (network for +2 reading frames).

\begin{tabular}{|c|c|c|c|c|}
\hline Sequence ID & In-degree & Out-degree & Degree & Cluster \\
\hline 2 - BetaCov/Wuhan/WHO01/2019 & 1 & 31 & 32 & $\overline{0}$ \\
\hline $6-\mathrm{BetaCoV} /$ Wuhan/WIV02/2019 & 2 & 28 & 30 & 0 \\
\hline 7 - BetaCoV/Wuhan/WIV05/2019 & 3 & 24 & 27 & 0 \\
\hline 8 - BetaCoV/Wuhan/WIV06/2019 & 4 & 22 & 26 & 0 \\
\hline 9 - BetaCoV/Wuhan/WIV07/2019 & 5 & 23 & 28 & 0 \\
\hline 16 - BetaCov/Wuhan/WH03/2020 & 7 & 23 & 30 & 0 \\
\hline 19 - BetaCov/Wuhan/WH04/2020 & 9 & 25 & 34 & 0 \\
\hline $21-\mathrm{BetaCoV} /$ Shenzhen/HKU-SZ-002/2020 & 10 & 20 & 30 & 0 \\
\hline 32 - BetaCoV/Zhejiang/WZ-0 1/2020 & 11 & 14 & 25 & 0 \\
\hline $33-$ BetaCoV/Shenzhen/SZTH-003/2020 & 16 & 19 & 35 & 0 \\
\hline $34-$ BetaCoV/Shenzhen/SZTH-004/2020 & 14 & 20 & 34 & 0 \\
\hline $36-$ BetaCoV/Zhejiang/WZ-02/2020 & 14 & 19 & 33 & 0 \\
\hline 42 - BetaCoV/Hangzhou/HZCDC000 1/2020 & 16 & 18 & 34 & 0 \\
\hline $45-$ BetaCoV/Zhejiang/Hangzhou- $1 / 2020$ & 16 & 16 & 32 & 0 \\
\hline $55-$ BetaCoV/Taiwan/2/2020 & 21 & 15 & 36 & 0 \\
\hline $59-$ BetaCoV/France/IDF0373/2020 & 14 & 12 & 26 & 0 \\
\hline $62-$ BetaCoV/Australia/NS WO $1 / 2020$ & 22 & 5 & 27 & 0 \\
\hline $64-$ BetaCoV/Japan/AI/I-004/2020 & 22 & 8 & 30 & 0 \\
\hline $65-\mathrm{BetaCoV} /$ Korea/KCDC03/2020 & 23 & 6 & 29 & 0 \\
\hline $69-$ BetaCoV/Germany/BavPat $1 / 2020$ & 6 & 7 & 13 & 0 \\
\hline 70 - BetaCoV/Australia/QLD0O1/2020 & 10 & 6 & 16 & 0 \\
\hline $79-$ BetaCoV/Australia/QLD02/2020 & 28 & 3 & 31 & 0 \\
\hline $80-$ BetaCoV/Taiwan/NTU01/2020 & 27 & 2 & 29 & 0 \\
\hline $81-$ BetaCoV/Singapore/3/2020 & 26 & 1 & 27 & 0 \\
\hline $1-\mathrm{BetaCoV} /$ Wuhan/IPBCAMS-WH-01/2019 & 0 & 3 & 3 & 1 \\
\hline 3 - BetaCoV/Wuhan/IV DC-HB-01/2019 & 2 & 2 & 4 & 1 \\
\hline 4- BetaCoV/Wuhan/IV DC-HB-05/2019 & 1 & 1 & 2 & 1 \\
\hline $5-\mathrm{BetaCoV} /$ Wuhan/WIV04/2019 & 2 & 6 & 8 & 1 \\
\hline 11 - BetaCoV/Wuhan/IPBCAMS-WH-04/2019 & 6 & 2 & 8 & 1 \\
\hline 12 - BetaCoV/Wuhan/IPBCAMS-WH-03/2019 & 2 & 3 & 5 & 1 \\
\hline 13 - BetaCoV/Wuhan/IPBCAMS-WH-02/2019 & 2 & 3 & 5 & 1 \\
\hline $14-\mathrm{BetaCoV} /$ Wuhan/IV DC-HB-04/2020 & 2 & 3 & 5 & 1 \\
\hline $15-\mathrm{BetaCoV} / \mathrm{Wuhan} / \mathrm{IPBCAMS}-\mathrm{WH}-05 / 2020$ & 2 & 7 & 9 & 1 \\
\hline 17 - BetaCoV/China/WHU01/2020 & 8 & 1 & 9 & 2 \\
\hline 18 - BetaCoV/China/WHU02/2020 & 1 & 13 & 14 & 2 \\
\hline 40 - BetaCoV/Beijing/IV DC-BJ-005/2020 & 12 & 9 & 21 & 2 \\
\hline $44-$ BetaCoV/Jiangsu/IVDC-JS-00 $1 / 2020$ & 6 & 11 & 17 & 2 \\
\hline $68-\mathrm{BetaCoV} /$ Singapore $/ 2 / 2020$ & 14 & 4 & 18 & 2 \\
\hline 77 - BetaCo V/France/IDF05 15/2020 & 7 & 5 & 12 & 2 \\
\hline $10-$ BetaCoV/Wuhan/HBCDC-HB-01/2019 & 6 & 6 & 12 & 3 \\
\hline $20-$ BetaCoV/Nonthaburi/6 $1 / 2020$ & 6 & 4 & 10 & 3 \\
\hline $24-$ BetaCoV/Nonthaburi/74/2020 & 3 & 4 & 7 & 3 \\
\hline $27-$ BetaCoV/Guangdong/20SFO0 12/2020 & 3 & 1 & 4 & 3 \\
\hline 28 - BetaCoV/Guangdong/20SFOO $13 / 2020$ & 1 & 2 & 3 & 3 \\
\hline 29 - BetaCoV/Guangdong/2OSFOO $14 / 2020$ & 2 & 3 & 5 & 3 \\
\hline $30-$ BetaCoV/Guangdong/20SF025/2020 & 2 & 6 & 8 & 3 \\
\hline 35 - BetaCoV/Guangdong/20SF028/2020 & 6 & 3 & 9 & 3 \\
\hline $38-$ BetaCoV/Guangdong/20SF040/2020 & 3 & 10 & 13 & 3 \\
\hline 58 - BetaCo V/France/IDF0372/2020 & 12 & 9 & 21 & 3 \\
\hline 78 - BetaCov/France/IDF0626/2020 & 11 & 4 & 15 & 3 \\
\hline $22-$ BetaCoV/Shenzhen/HKU-SZ-005/2020 & 8 & 4 & 12 & 4 \\
\hline $23-\mathrm{BetaCoV} / J i a n g x i / I V$ DC-JX-002/2020 & 4 & 5 & 9 & 4 \\
\hline $25-$ BetaCoV/Shenzhen/SZTH-00 $1 / 2020$ & 7 & 5 & 12 & 4 \\
\hline $26-$ BetaCoV/Shenzhen/SZTH-002/2020 & 3 & 6 & 9 & 4 \\
\hline $31-$ BetaCoV/Sichuan/ITVDC-SC-00 1/2020 & 4 & 9 & 13 & 4 \\
\hline $37-$ BetaCoV/Yunnan/IVDC- Y N-003/2020 & 8 & 3 & 11 & 4 \\
\hline 39 - BetaCoV/Chongqing/IVDC-CQ-00 $1 / 2020$ & 4 & 3 & 7 & 4 \\
\hline $41-\mathrm{BetaCoV} / \mathrm{USA} / \mathrm{WA} 1 / 2020$ & 5 & 5 & 10 & 4 \\
\hline $43-$ BetaCoV/Shandong/IVDC-SD-00 $1 / 2020$ & 6 & 7 & 13 & 4 \\
\hline $46-\mathrm{BetaCoV} / \mathrm{USA} / \mathrm{IL} 1 / 2020$ & 5 & 3 & 8 & 4 \\
\hline $50-$ BetaCoV/Guangdong/20SF $174 / 2020$ & 19 & 2 & 21 & 5 \\
\hline $51-$ BetaCoV/Guangzhou/20SF 206/2020 & 7 & 3 & 10 & 5 \\
\hline 52 - BetaCo V/Foshan/20SF207/2020 & 2 & 2 & 4 & 5 \\
\hline 53 - BetaCo V/Foshan/20SF2 10/2020 & 2 & 2 & 4 & 5 \\
\hline $54-$ BetaCoV/Foshan/20SF21 $1 / 2020$ & 1 & 3 & 4 & 5 \\
\hline $57-$ BetaCoV/Guangdong/20SF 201/2020 & 5 & 12 & 17 & 5 \\
\hline 47 - BetaCoV/Chonggqing/Y CO $1 / 2020$ & 20 & 9 & 29 & 6 \\
\hline $48-\mathrm{BetaCoV} / \mathrm{USA} / \mathrm{CA} 2 / 2020$ & 4 & 4 & 8 & 6 \\
\hline $49-\mathrm{BetaCoV} / \mathrm{USA} / \mathrm{AZ} 1 / 2020$ & 6 & 5 & 11 & 6 \\
\hline $56-\mathrm{BetaCoV} / \mathrm{USA} / \mathrm{CA} 1 / 2020$ & 6 & 7 & 13 & 6 \\
\hline $60-$ BetaCoV/Singapore/ 1/2020 & 6 & 5 & 11 & 6 \\
\hline $61-$ BetaCoV/Chonggqing/ZX0 $1 / 2020$ & 7 & 10 & 17 & 6 \\
\hline 63 - BetaCoV/Australia/VICO1/2020 & 15 & 7 & 22 & 7 \\
\hline $66-\mathrm{BetaCoV} / \mathrm{USA} / \mathrm{WA} 1-\mathrm{A} 12 / 2020$ & 7 & 1 & 8 & 7 \\
\hline $67-\mathrm{BetaCoV} / \mathrm{USA} / \mathrm{W}$ A $1-\mathrm{F} 6 / 2020$ & 1 & 8 & 9 & 7 \\
\hline $71-$ BetaCoV/England/01/2020 & 6 & 2 & 8 & 7 \\
\hline $72-$ BetaCoV/England $/ 02 / 2020$ & 2 & 3 & 5 & 7 \\
\hline 73 - BetaCoV/Finland $/ 1 / 2020$ & 4 & 2 & 6 & 7 \\
\hline $74-\mathrm{BetaCoV} / \mathrm{USA} / \mathrm{CA} 3 / 2020$ & 4 & 1 & 5 & 7 \\
\hline $75-\mathrm{BetaCoV} / \mathrm{USA} / \mathrm{CA} 4 / 2020$ & 1 & 2 & 3 & 7 \\
\hline $76-\mathrm{BetaCoV} / \mathrm{USA} / \mathrm{CA} 5 / 2020$ & 2 & 6 & 8 & 7 \\
\hline $82-$ BetaCoV/Belgium/GHB-0302 1/2020 & 8 & 0 & 8 & 7 \\
\hline
\end{tabular}

Table 16. Intra-cluster similarities (network for +2 reading frames).

\begin{tabular}{c|c|c|c|c|c} 
Cluster & Avg. similarity & SD & Min. similarity & Max. similarity & Cluster size \\
\hline \hline 0 & 0.277353 & 0.088247 & 0.250620 & 0.999866 & 24 \\
1 & 0.999730 & 0.000222 & 0.999231 & 1.000000 & 9 \\
2 & 0.998768 & 0.001345 & 0.996387 & 1.000000 & 6 \\
3 & 0.999915 & 0.000048 & 0.999832 & 1.000000 & 11 \\
4 & 0.999385 & 0.000371 & 0.998461 & 0.999933 & 10 \\
5 & 0.999926 & 0.000036 & 0.999866 & 1.000000 & 6 \\
6 & 0.883701 & 0.112398 & 0.781976 & 0.999899 & 6 \\
7 & 0.997900 & 0.002419 & 0.991158 & 1.000000 & 10
\end{tabular}


medRxiv preprint doi: https://doi.org/10.1101/2020.02.07.20021139; this version posted February 17, 2020. The copyright holder for this preprint (which was not certified by peer review) is the author/funder, who has granted medRxiv a license to display the preprint in perpetuity.

It is made available under a CC-BY 4.0 International license .

Figure 8. +3 reading frame network. 
medRxiv preprint doi: https://doi.org/10.1101/2020.02.07.20021139; this version posted February 17, 2020. The copyright holder for this preprint (which was not certified by peer review) is the author/funder, who has granted medRxiv a license to display the preprint in perpetuity. It is made available under a CC-BY 4.0 International license .

Table 17. Cluster memberships of the nucleotide sequences (network for +3 reading frames).

\begin{tabular}{|c|c|c|c|c|}
\hline Sequence ID & In-degree & Out-degree & Degree & Cluster \\
\hline 2 - BetaCov/Wuhan/WHO01/2019 & 1 & 31 & 32 & 0 \\
\hline 6- BetaCoV/Wuhan/WIV02/2019 & 2 & 25 & 27 & 0 \\
\hline 7 - BetaCoV/Wuhan/WIV05/2019 & 3 & 25 & 28 & 0 \\
\hline 8 - BetaCoV/Wuhan/WIV06/2019 & 4 & 24 & 28 & 0 \\
\hline 9 - BetaCoV/Wuhan/WIV07/2019 & 5 & 24 & 29 & 0 \\
\hline 16 - BetaCov/Wuhan/WH03/2020 & 7 & 23 & 30 & 0 \\
\hline 19 - BetaCov/Wuhan/WH04/2020 & 9 & 23 & 32 & 0 \\
\hline $21-$ BetaCoV/Shenzhen/HKU-SZ-002/2020 & 10 & 19 & 29 & 0 \\
\hline $32-$ BetaCoV/Zhejiang/WZ-0 1/2020 & 13 & 13 & 26 & 0 \\
\hline $33-\mathrm{BetaCoV} /$ Shenzhen/SZTH-003/2020 & 16 & 18 & 34 & 0 \\
\hline $34-$ BetaCoV/Shenzhen/SZTH-004/2020 & 13 & 17 & 30 & 0 \\
\hline $36-$ BetaCoV/Zhejiang/WZ-02/2020 & 17 & 16 & 33 & 0 \\
\hline 42 - BetaCoV/Hangzhou/HZCDC000 1/2020 & 16 & 14 & 30 & 0 \\
\hline $45-$ BetaCoV/Zhejiang/Hangzhou- $1 / 2020$ & 18 & 15 & 33 & 0 \\
\hline $55-$ BetaCoV/Taiwan $/ 2 / 2020$ & 20 & 13 & 33 & 0 \\
\hline $59-$ BetaCoV/France/IDF0373/2020 & 14 & 10 & 24 & 0 \\
\hline $62-$ BetaCoV/Australia/NS WO 1/2020 & 22 & 5 & 27 & 0 \\
\hline $64-$ BetaCoV/Japan/AI/I-004/2020 & 22 & 8 & 30 & 0 \\
\hline $65-\mathrm{BetaCoV} /$ Korea/KCDC03/2020 & 22 & 5 & 27 & 0 \\
\hline $69-$ BetaCoV/Germany/BavPat $1 / 2020$ & 8 & 7 & 15 & 0 \\
\hline 70 - BetaCoV/Australia/QLD0O1/2020 & 10 & 6 & 16 & 0 \\
\hline 79 - BetaCoV/Australia/QLD02/2020 & 24 & 3 & 27 & 0 \\
\hline $80-$ BetaCoV/Taiwan/NTU01/2020 & 27 & 2 & 29 & 0 \\
\hline $81-$ BetaCoV/Singapore/3/2020 & 25 & 1 & 26 & 0 \\
\hline 1 - BetaCoV/Wuhan/IPBCAMS-WH-01/2019 & 0 & 3 & 3 & 1 \\
\hline $3-\mathrm{BetaCoV} /$ Wuhan/IV DC-HB-01/2019 & 2 & 2 & 4 & 1 \\
\hline 4 - BetaCoV/Wuhan/IV DC-HB-05/2019 & 1 & 2 & 3 & 1 \\
\hline 5 - BetaCoV/Wuhan/WIV04/2019 & 2 & 7 & 9 & 1 \\
\hline $11-\mathrm{BetaCoV} /$ Wuhan/IPBCAMS-WH-04/2019 & 6 & 2 & 8 & 1 \\
\hline $12-\mathrm{BetaCoV} / \mathrm{Wuhan} / \mathrm{IPBCAMS}-\mathrm{WH}-03 / 2019$ & 3 & 3 & 6 & 1 \\
\hline $13-\mathrm{BetaCoV} / \mathrm{Wuhan} / \mathrm{IPBCAMS}-\mathrm{WH}-02 / 2019$ & 3 & 3 & 6 & 1 \\
\hline $14-\mathrm{BetaCoV} / \mathrm{Wuhan} / \mathrm{IV}$ DC-HB-04/2020 & 2 & 3 & 5 & 1 \\
\hline $15-\mathrm{BetaCoV} / \mathrm{Wuhan} / \mathrm{IPBCAMS}-\mathrm{WH}-05 / 2020$ & 3 & 8 & 11 & 1 \\
\hline $17-\mathrm{BetaCoV} /$ China/WHU01/2020 & 8 & 1 & 9 & 2 \\
\hline 18 - BetaCoV/China/WHU02/2020 & 1 & 11 & 12 & 2 \\
\hline $40-$ BetaCoV/Beijing/IV DC-BJ-005/2020 & 12 & 9 & 21 & 2 \\
\hline $44-\mathrm{BetaCoV} / J i a n g s u / I V D C-J S-001 / 2020$ & 6 & 11 & 17 & 2 \\
\hline $68-\mathrm{BetaCoV} /$ Singapore/2/2020 & 13 & 5 & 18 & 2 \\
\hline 77 - BetaCo V/France/IDF05 15/2020 & 7 & 5 & 12 & 2 \\
\hline $22-$ BetaCoV/Shenzhen/HKU-SZ-005/2020 & 8 & 4 & 12 & 3 \\
\hline $23-$ BetaCoV/Jiangxi/IV DC-JX-002/2020 & 2 & 6 & 8 & 3 \\
\hline $25-$ BetaCoV/Shenzhen/SZTH-00 1/2020 & 4 & 5 & 9 & 3 \\
\hline $26-$ BetaCoV/Shenzhen/SZTH-002/2020 & 3 & 7 & 10 & 3 \\
\hline $31-$ BetaCoV/Sichuan/ITVDC-SC-00 1/2020 & 4 & 8 & 12 & 3 \\
\hline $37-$ BetaCoV/Yunnan/IVDC- Y N-003/2020 & 7 & 3 & 10 & 3 \\
\hline $39-$ BetaCoV/Chongqing/IVDC-CQ-00 $1 / 2020$ & 4 & 3 & 7 & 3 \\
\hline $41-\mathrm{BetaCoV} / \mathrm{USA} / \mathrm{WA} 1 / 2020$ & 5 & 6 & 11 & 3 \\
\hline $43-$ BetaCoV/Shandong/IVDC-SD-00 1/2020 & 5 & 7 & 12 & 3 \\
\hline $10-$ BetaCoV/Wuhan/HBCDC-HB-01/2019 & 6 & 5 & 11 & 4 \\
\hline $20-\mathrm{BetaCoV} /$ Nonthaburi/6 1/2020 & 5 & 3 & 8 & 4 \\
\hline 24 - BetaCoV/Nonthaburi $/ 74 / 2020$ & 3 & 4 & 7 & 4 \\
\hline 27 - BetaCoV/Guangdong/20SFOO 12/2020 & 2 & 1 & 3 & 4 \\
\hline 28 - BetaCoV/Guangdong/20SFOO 13/2020 & 1 & 2 & 3 & 4 \\
\hline 29 - BetaCoV/Guangdong/2OSFOO $14 / 2020$ & 2 & 2 & 4 & 4 \\
\hline $30-$ BetaCoV/Guangdong/20SF025/2020 & 2 & 7 & 9 & 4 \\
\hline 35 - BetaCoV/Guangdong/20SF028/2020 & 6 & 3 & 9 & 4 \\
\hline 38 - BetaCoV/Guangdong/20SF 040/2020 & 3 & 11 & 14 & 4 \\
\hline 58 - BetaCo V/France/IDF0372/2020 & 12 & 12 & 24 & 4 \\
\hline 78 - BetaCov/France/IDF0626/2020 & 10 & 4 & 14 & 4 \\
\hline $46-\mathrm{BetaCoV} / \mathrm{USA} / \mathrm{IL} 1 / 2020$ & 4 & 3 & 7 & 5 \\
\hline 47 - BetaCoV/Chonggqing/Y C0 $1 / 2020$ & 20 & 9 & 29 & 5 \\
\hline $48-\mathrm{BetaCoV} / \mathrm{USA} / \mathrm{CA} 2 / 2020$ & 3 & 5 & 8 & 5 \\
\hline $49-\mathrm{BetaCoV} / \mathrm{USA} / \mathrm{AZ} 1 / 2020$ & 6 & 5 & 11 & 5 \\
\hline $56-\mathrm{BetaCoV} / \mathrm{USA} / \mathrm{CA} 1 / 2020$ & 5 & 7 & 12 & 5 \\
\hline $60-$ BetaCoV/Singapore/ 1/2020 & 7 & 4 & 11 & 5 \\
\hline 61 - BetaCoV/Chonggqing/ZX0 $1 / 2020$ & 7 & 12 & 19 & 5 \\
\hline $50-$ BetaCoV/Guangdong/20SF $174 / 2020$ & 18 & 2 & 20 & 6 \\
\hline 51 - BetaCoV/Guangzhou/20SF206/2020 & 5 & 2 & 7 & 6 \\
\hline 52 - BetaCo V/Foshan/20SF 207/2020 & 2 & 3 & 5 & 6 \\
\hline 53 - BetaCo V/Foshan/20SF2 10/2020 & 2 & 2 & 4 & 6 \\
\hline $54-\mathrm{BetaCoV} /$ Foshan/20SF 21 1/2020 & 1 & 3 & 4 & 6 \\
\hline 57 - BetaCoV/Guangdong/20SF $201 / 2020$ & 5 & 13 & 18 & 6 \\
\hline $63-$ BetaCoV/Australia/VICO1/2020 & 14 & 8 & 22 & 7 \\
\hline $66-\mathrm{BetaCoV} / \mathrm{USA} / \mathrm{WA} 1-\mathrm{A} 12 / 2020$ & 8 & 1 & 9 & 7 \\
\hline $67-\mathrm{BetaCoV} / \mathrm{USA} / \mathrm{W}$ A $1-\mathrm{F} 6 / 2020$ & 1 & 6 & 7 & 7 \\
\hline $71-$ BetaCoV/England/01/2020 & 5 & 2 & 7 & 7 \\
\hline $72-$ BetaCoV/England $/ 02 / 2020$ & 3 & 3 & 6 & 7 \\
\hline $73-$ BetaCoV/Finland/1/2020 & 4 & 2 & 6 & 7 \\
\hline $74-\mathrm{BetaCoV} / \mathrm{USA} / \mathrm{CA} 3 / 2020$ & 3 & 1 & 4 & 7 \\
\hline $75-\mathrm{BetaCoV} / \mathrm{USA} / \mathrm{CA} 4 / 2020$ & 1 & 2 & 3 & 7 \\
\hline $76-\mathrm{BetaCoV} / \mathrm{USA} / \mathrm{CA} 5 / 2020$ & 3 & 6 & 9 & 7 \\
\hline $82-$ BetaCoV/Belgium/GHB-0302 1/2020 & 8 & 0 & 8 & 7 \\
\hline
\end{tabular}

Table 18. Intra-cluster similarities (network for +3 reading frames).

\begin{tabular}{c|c|c|c|c|c} 
Cluster & Avg. similarity & SD & Min. similarity & Max. similarity & Cluster size \\
\hline \hline 0 & 0.277353 & 0.088247 & 0.250620 & 0.999866 & 24 \\
1 & 0.999730 & 0.000222 & 0.999231 & 1.000000 & 9 \\
2 & 0.998768 & 0.001345 & 0.996387 & 1.000000 & 6 \\
3 & 0.999388 & 0.000391 & 0.998461 & 0.999933 & 9 \\
4 & 0.999915 & 0.000048 & 0.999832 & 1.000000 & 11 \\
5 & 0.896098 & 0.111390 & 0.781909 & 0.999899 & 7 \\
6 & 0.999926 & 0.000036 & 0.999866 & 1.000000 & 6 \\
7 & 0.997900 & 0.002419 & 0.991158 & 1.000000 & 10
\end{tabular}




\section{Acknowledgements}

We want to thank the laboratories and researchers who contributed the genomic research data used in this study and obtained via the GISAID EpiFlu Database ${ }^{T M}{ }^{3}$ on Monday, February 10th 2020. The following tables contain information about the origination of the research data used in this study and give credit to the collecting and submitting researchers and laboratories.

$\sqrt[3]{\text { https://www.gisaid.org/ }}$ 
Table 19. Nucleotide sequences used in this study that were obtained from the GISAID EpiFlu Database ${ }^{T M}$.

\begin{tabular}{|c|c|c|c|c|}
\hline Collection date & Sequence id & Originating lab & Submitting lab & Authors \\
\hline $24 / 12 / 19$ & $\begin{array}{l}\text { BetaCoV/Wuhan/IPBCAMS-WH- } \\
01 / 2019\end{array}$ & $\begin{array}{l}\text { Institute of Pathogen } \\
\text { Biology }\end{array}$ & 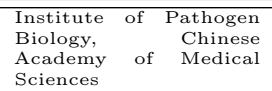 & $\begin{array}{l}\text { Lili Ren, Jianwei Wang, Qi Jin, Zichun } \\
\text { Xiang, Zhigiang Wu, Chao Wu, Yiwei } \\
\text { Liu }\end{array}$ \\
\hline $26 / 12 / 19$ & BetaCov/Wuhan/WHO01/2019 & $\begin{array}{l}\text { General Hospital of } \\
\text { Central Theater Com- } \\
\text { mand of People }\end{array}$ & BGI & $\begin{array}{l}\text { Weijun Chen, Yuhai Bi, Weifeng Shi } \\
\text { and Zhenhong Hu }\end{array}$ \\
\hline $30 / 12 / 19$ & BetaCoV/Wuhan/IV DC-HB-01/2019 & $\begin{array}{l}\text { National Institute for } \\
\text { Viral Disease Control } \\
\text { and Prevention }\end{array}$ & $\begin{array}{l}\text { National Institute for } \\
\text { Viral Disease Control } \\
\text { and Prevention, China } \\
\text { CDC }\end{array}$ & $\begin{array}{l}\text { Wenjie TanXiang ZhaoWenling Wang } \\
\text { Xuejun MaYongzhong JiangRoujian } \\
\text { Lu, Ji Wang, Weimin ZhouPeihua } \\
\text { NiuPeipei LiuFaxian ZhanWeifeng } \\
\text { ShiBaoying HuangJun LiuLi ZhaoYao } \\
\text { MengXiaozhou HeFei YeNa ZhuYang } \\
\text { LiJing ChenWenbo XuGeorge F. } \\
\text { GaoGuizhen Wu }\end{array}$ \\
\hline $30 / 12 / 19$ & BetaCoV/Wuhan/IV DC-HB-05/2019 & $\begin{array}{l}\text { National Institute for } \\
\text { Viral Disease Control } \\
\text { and Prevention }\end{array}$ & $\begin{array}{l}\text { National Institute for } \\
\text { Viral Disease Control } \\
\text { and Prevention, China } \\
\text { CDC }\end{array}$ & $\begin{array}{l}\text { Wenjie TanXuejun MaXiang ZhaoWen- } \\
\text { ling Wang Yongzhong JiangRoujian } \\
\text { LuJi WangPeihua Niu, Weimin Zhou, } \\
\text { Faxian ZhanWeifeng ShiBaoying } \\
\text { HuangJun LiuLi ZhaoYao MengFei } \\
\text { YeNa Zhu, Xiaozhou HePeipei Liu, } \\
\text { Yang LiJing ChenWenbo XuGeorge F. }\end{array}$ \\
\hline $30 / 12 / 19$ & BetaCoV/Wuhan/WIV04/2019 & $\begin{array}{l}\text { Wuhan Jinyintan Hospi- } \\
\text { tal }\end{array}$ & $\begin{array}{l}\text { Wuhan Institute of } \\
\text { Virology, Chinese } \\
\text { Academy of Sciences }\end{array}$ & $\begin{array}{l}\text { Peng Zhou, Xing-Lou Yang, Ding-Yu } \\
\text { Zhang, Lei Zhang, Yan Zhu, Hao-Rui } \\
\text { Si, Zhengli Shi }\end{array}$ \\
\hline $30 / 12 / 19$ & BetaCoV/Wuhan/WIV05/2019 & $\begin{array}{l}\text { Wuhan Jinyintan Hospi- } \\
\text { tal }\end{array}$ & $\begin{array}{l}\text { Wuhan Institute of } \\
\text { Virology, } \begin{array}{c}\text { Chinese } \\
\text { Academy of Sciences }\end{array}\end{array}$ & $\begin{array}{l}\text { Peng Zhou, Xing-Lou Yang, Ding-Yu } \\
\text { Zhang, Lei Zhang, Yan Zhu, Hao-Rui } \\
\text { Si, Zhengli Shi }\end{array}$ \\
\hline $30 / 12 / 19$ & BetaCoV/Wuhan/WIV06/2019 & $\begin{array}{l}\text { Wuhan Jinyintan Hospi- } \\
\text { tal }\end{array}$ & $\begin{array}{l}\text { Wuhan Institute of } \\
\text { Virology, } \quad \text { Chinese } \\
\text { Academy of Sciences }\end{array}$ & $\begin{array}{l}\text { Peng Zhou, Xing-Lou Yang, Ding-Yu } \\
\text { Zhang, Lei Zhang, Yan Zhu, Hao-Rui } \\
\text { Si, Zhengli Shi }\end{array}$ \\
\hline $30 / 12 / 19$ & BetaCoV/Wuhan/WIV07/2019 & $\begin{array}{l}\text { Wuhan Jinyintan Hospi- } \\
\text { tal }\end{array}$ & $\begin{array}{l}\text { Wuhan Institute of } \\
\text { Virology, } \\
\text { Academy of Sciences }\end{array}$ & $\begin{array}{l}\text { Peng Zhou, Xing-Lou Yang, Ding-Yu } \\
\text { Zhang, Lei Zhang, Yan Zhu, Hao-Rui } \\
\text { Si, Zhengli Shi }\end{array}$ \\
\hline $30 / 12 / 19$ & $\begin{array}{l}\text { BetaCoV/Wuhan/HBCDC-HB- } \\
01 / 2019\end{array}$ & $\begin{array}{l}\text { Wuhan Jinyintan Hospi- } \\
\text { tal }\end{array}$ & $\begin{array}{l}\text { Hubei Provincial Center } \\
\text { for Disease Control and } \\
\text { Prevention }\end{array}$ & $\begin{array}{l}\text { Bin Fang, Xiang Li, Xiao Yu, Linlin } \\
\text { Liu, Bo Yang, Faxian Zhan, Guojun } \\
\text { Ye, Xixiang Huo, Jungiang Xu, Bo Yu, } \\
\text { Kun Cai, Jing Li, Yongzhong Jiang. }\end{array}$ \\
\hline $30 / 12 / 19$ & $\begin{array}{l}\text { BetaCoV/Wuhan/IPBCAMS-WH- } \\
\text { 04/2019 }\end{array}$ & $\begin{array}{l}\text { Institute of Pathogen } \\
\text { Biology }\end{array}$ & $\begin{array}{l}\text { Institute of } \begin{array}{r}\text { Pathogen } \\
\text { Chinese }\end{array} \\
\text { Acadogy, of } \\
\text { Sciences }\end{array}$ & $\begin{array}{l}\text { Lili Ren, Jianwei Wang, Qi Jin, Zichun } \\
\text { Xiang, Zhigiang Wu, Chao Wu, Yiwei } \\
\text { Liu }\end{array}$ \\
\hline $30 / 12 / 19$ & $\begin{array}{l}\text { BetaCoV/Wuhan/IPBCAMS-WH- } \\
\text { 03/2019 }\end{array}$ & $\begin{array}{l}\text { Institute of Pathogen } \\
\text { Biology }\end{array}$ & 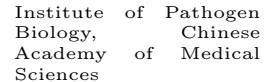 & $\begin{array}{l}\text { Lili Ren, Jianwei Wang, Qi Jin, Zichun } \\
\text { Xiang, Zhigiang Wu, Chao Wu, Yiwei } \\
\text { Liu }\end{array}$ \\
\hline $30 / 12 / 19$ & $\begin{array}{l}\text { BetaCoV/Wuhan/IPBCAMS-WH- } \\
02 / 2019\end{array}$ & $\begin{array}{l}\text { Institute of Pathogen } \\
\text { Biology }\end{array}$ & $\begin{array}{l}\text { Institute of Pathogen } \\
\text { Biology, } \\
\text { Academy of } \begin{array}{l}\text { Medinese } \\
\text { Sciences }\end{array}\end{array}$ & $\begin{array}{l}\text { Lili Ren, Jianwei Wang, Qi Jin, Zichun } \\
\text { Xiang, Zhigiang Wu, Chao Wu, Yiwei } \\
\text { Liu }\end{array}$ \\
\hline $1 / 01 / 20$ & BetaCoV/Wuhan/IV DC-HB-04/2020 & $\begin{array}{l}\text { National Institute for } \\
\text { Viral Disease Control } \\
\text { and Prevention }\end{array}$ & $\begin{array}{l}\text { National Institute for } \\
\text { Viral Disease Control } \\
\text { and Prevention, China } \\
\text { CDC }\end{array}$ & $\begin{array}{l}\text { Wenjie TanXiang ZhaoWenling } \\
\text { WangXuejyun MaYongzhong Jian- } \\
\text { gRoujian LuJi WangWeimin ZhouPei- } \\
\text { hua NiuPeipei LiuFaxian ZhanWeifeng } \\
\text { ShiBaoying HuangJun LiuLi ZhaoYao } \\
\text { MengXiaozhou HeFei YeNa ZhuYang } \\
\text { LiJing ChenWenbo XuGeorge F. } \\
\text { GaoGuizhen Wu }\end{array}$ \\
\hline $1 / 01 / 20$ & $\begin{array}{l}\text { BetaCoV/Wuhan/IPBCAMS-WH- } \\
05 / 2020\end{array}$ & $\begin{array}{l}\text { Institute of Pathogen } \\
\text { Biology }\end{array}$ & $\begin{array}{l}\text { Institute of } \begin{array}{r}\text { Pathogen } \\
\text { Biology, } \\
\text { Academy of } \\
\text { Sciences }\end{array} \text { Medical } \\
\text { Sciences }\end{array}$ & $\begin{array}{l}\text { Lili Ren, Jianwei Wang, Qi Jin, Zichun } \\
\text { Xiang, Zhigiang Wu, Chao Wu, Yiwei } \\
\text { Liu }\end{array}$ \\
\hline $1 / 01 / 20$ & BetaCov/Wuhan/WH03/2020 & $\begin{array}{l}\text { General Hospital of } \\
\text { Central Theater Com- } \\
\text { mand of People }\end{array}$ & BGI & $\begin{array}{l}\text { Weijun Chen, Yuhai Bi, Weifeng Shi } \\
\text { and Zhenhong Hu }\end{array}$ \\
\hline $2 / 01 / 20$ & BetaCoV/China/WHU01/2020 & unknown & $\begin{array}{l}\text { State Key Laboratory } \\
\text { of Virology, Wuhan Uni- } \\
\text { versity }\end{array}$ & $\begin{array}{l}\text { Chen,L., Liu,W., Zhang,Q., Xu,K., } \\
\text { Ye,G., Wu,W., Sun,Z., Liu,F., Wu,K., } \\
\text { Mei, Y., Zhang,W., Chen, Y., Li,Y., } \\
\text { Shi,M., Lan,K. and Liu,Y. }\end{array}$ \\
\hline $2 / 01 / 20$ & BetaCoV/China/WHU02/2020 & unknown & $\begin{array}{l}\text { State Key Laboratory } \\
\text { of Virology, Wuhan Uni- } \\
\text { versity }\end{array}$ & $\begin{array}{l}\text { Chen,L., Liu,W., Zhang,Q., Xu,K., } \\
\text { Ye,G., Wu,W., Sun,Z., Liu,F., Wu,K., } \\
\text { Mei, Y., Zhang,W., Chen, Y., Li,Y., } \\
\text { Shi,M., Lan,K. and Liu,Y. }\end{array}$ \\
\hline $5 / 01 / 20$ & BetaCov/Wuhan/WH04/2020 & $\begin{array}{l}\text { General Hospital of } \\
\text { Central Theater Com- } \\
\text { mand of People }\end{array}$ & BGI & $\begin{array}{l}\text { Weijun Chen, Yuhai Bi, Weifeng Shi } \\
\text { and Zhenhong } \mathrm{Hu}\end{array}$ \\
\hline $8 / 01 / 20$ & BetaCoV/Nonthaburi/6 1/2020 & $\begin{array}{l}\text { Bamrasnaradura Hospi- } \\
\text { tal }\end{array}$ & $\begin{array}{l}\text { 1. Department of Med- } \\
\text { ical Sciences, Ministry } \\
\text { of Public Health, Thai- } \\
\text { land }\end{array}$ & NA \\
\hline $10 / 01 / 20$ & $\begin{array}{l}\text { BetaCoV/Shenzhen/HKU-SZ- } \\
002 / 2020\end{array}$ & $\begin{array}{l}\text { The University of Hong } \\
\text { Kong - Shenzhen Hospi- } \\
\text { tal }\end{array}$ & $\begin{array}{l}\text { Li Ka Shing Faculty of } \\
\text { Medicine, The Univer- } \\
\text { sity of Hong Kong }\end{array}$ & $\begin{array}{l}\text { Chan,J.F.-W., Yuan,S., Kok,K.H., } \\
\text { To,K.K.-W., Chu,H., Yang,J., Xing,F., } \\
\text { Liu,J., Yip,C.C.-Y., Poon,R.W-S., } \\
\text { Tsai,H.W., Lo,S.K.-F., Chan,K.H., } \\
\text { Poon,V.K.-M., Chan,W.M.., Ip,J.D., } \\
\text { Cai,J.P., Cheng,V.C.-C., Chen,H., } \\
\text { Hui,C.K.-M. and Yuen,K.Y. }\end{array}$ \\
\hline $11 / 01 / 20$ & $\begin{array}{l}\text { BetaCoV/Shenzhen/HKU-SZ- } \\
005 / 2020\end{array}$ & $\begin{array}{l}\text { The University of Hong } \\
\text { Kong - Shenzhen Hospi- } \\
\text { tal }\end{array}$ & $\begin{array}{l}\text { Li Ka Shing Faculty of } \\
\text { Medicine, The Univer- } \\
\text { sity of Hong Kong }\end{array}$ & $\begin{array}{l}\text { Chan,J.F.-W., Yuan,S., Kok,K.H., } \\
\text { To,K.K.-W., Chu,H., Yang,J., Xing,F., } \\
\text { Liu,J., Yip,C.C.-Y., Poon,R.W-S., } \\
\text { Tsai,H.W., Lo,S.K.-F., Chan,K.H., } \\
\text { Poon,V.K.-M., Chan,W.M.., Ip,J.D., } \\
\text { Cai,J.P., Cheng,V.C.-C., Chen,H., } \\
\text { Hui,C.K.-M. and Yuen,K.Y. }\end{array}$ \\
\hline $11 / 01 / 20$ & $\begin{array}{l}\text { BetaCoV/Jiangxi/IV } \\
002 / 2020\end{array}$ & $\begin{array}{l}\text { National Institute for } \\
\text { Viral Disease Control } \\
\text { and Prevention }\end{array}$ & $\begin{array}{l}\text { National Institute for } \\
\text { Viral Disease Control }\end{array}$ & $\begin{array}{l}\text { Wenjie Tan, Yong Shi, Wenling Wang, } \\
\text { Peihua Niu, Roujian Lu, Jianxiong Li, } \\
\text { Xiang Zhao, Baoying Huang, Li Zhao, } \\
\text { Fei Ye, Wenbo Xu, George F. Gao, } \\
\text { Guizhen Wu }\end{array}$ \\
\hline
\end{tabular}


medRxiv preprint doi: https://doi.org/10.1101/2020.02.07.20021139; this version posted February 17, 2020. The copyright holder for this preprint (which was not certified by peer review) is the author/funder, who has granted medRxiv a license to display the preprint in perpetuity.

It is made available under a CC-BY 4.0 International license .

Table 20. Nucleotide sequences used in this study that were obtained from the GISAID EpiFlu Database ${ }^{\mathrm{TM}}$ (contd.).

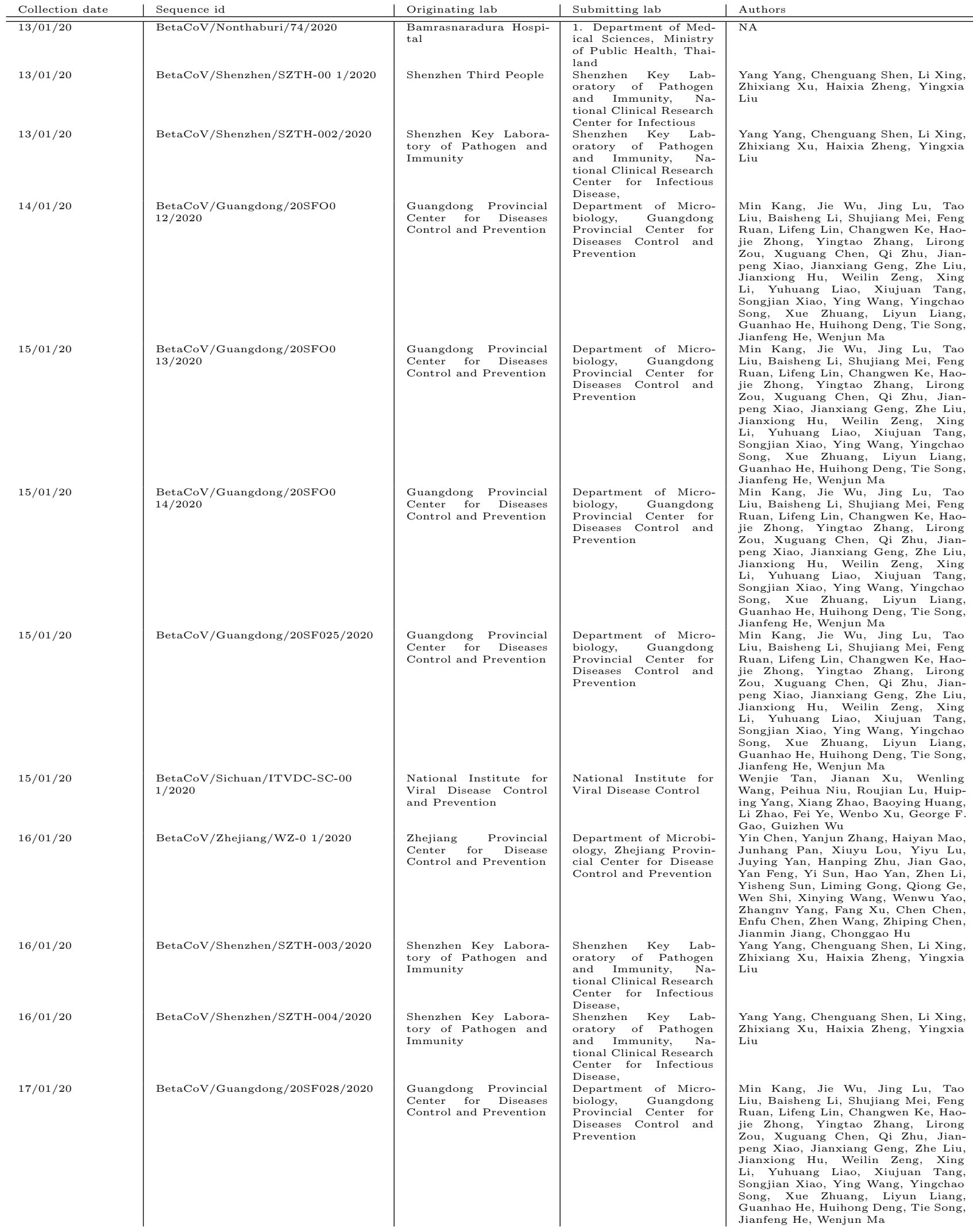


Table 21. Nucleotide sequences used in this study that were obtained from the GISAID EpiFlu Database ${ }^{\mathrm{TM}}$ (contd.).

\begin{tabular}{|c|c|c|c|c|}
\hline Collection date & Sequence id & Originating lab & Submitting lab & Authors \\
\hline $17 / 01 / 20$ & BetaCoV/Zhejiang/WZ-02/2020 & $\begin{array}{l}\text { Zhejiang } r \text { Provincial } \\
\text { Center for Disease } \\
\text { Control and Prevention }\end{array}$ & $\begin{array}{l}\text { Department of Microbi- } \\
\text { ology, Zhejiang Provin- } \\
\text { cial Center for Disease } \\
\text { Control and Prevention }\end{array}$ & $\begin{array}{l}\text { Yanjun Zhang, Yin Chen, Haiyan Mao, } \\
\text { Junhang Pan, Xiuyu Lou, Yiyu Lu, } \\
\text { Juying Yan, Hanping Zhu, Jian Gao, } \\
\text { Yan Feng, Yi Sun, Hao Yan, Zhen Li, } \\
\text { Yisheng Sun, Liming Gong, Qiong Ge, } \\
\text { Wen Shi, Xinying Wang, Wenwu Yao, } \\
\text { Zhangnv Yang, Fang Xu, Chen Chen, } \\
\text { Enfu Chen, Zhen Wang, Zhiping Chen, } \\
\text { Jianmin Jiang, Chonggao Hu }\end{array}$ \\
\hline $17 / 01 / 20$ & $\begin{array}{l}\text { BetaCoV/Yunnan/IVDC- } \\
003 / 2020\end{array}$ & $\begin{array}{l}\text { National Institute for } \\
\text { Viral Disease Control } \\
\text { and Prevention }\end{array}$ & $\begin{array}{l}\text { National Institute for } \\
\text { Viral Disease Control }\end{array}$ & $\begin{array}{l}\text { Wenjie TanXiaoging FuXiang } \\
\text { ZhaoWenling Wang Peihua Ni- } \\
\text { uRoujian Lu,Yanhong SunBaoying } \\
\text { HuangLi ZhaoFei YeWenbo XuGeorge } \\
\text { F. GaoGuizhen Wu }\end{array}$ \\
\hline $18 / 01 / 20$ & BetaCoV/Guangdong/20SF040/2020 & $\begin{array}{l}\text { Guangdong Provincial } \\
\text { Center for Piseases } \\
\text { Control and Prevention }\end{array}$ & $\begin{array}{l}\text { Department of Micro- } \\
\text { biology, Guangdong } \\
\text { Provincial Center for } \\
\text { Diseases Control and } \\
\text { Prevention }\end{array}$ & $\begin{array}{l}\text { Min Kang, Jie Wu, Jing Lu, Tao } \\
\text { Liu, Baisheng Li, Shujiang Mei, Feng } \\
\text { Ruan, Lifeng Lin, Changwen Ke, Hao- } \\
\text { jie Zhong, Yingtao Zhang, Lirong } \\
\text { Zou, Xuguang Chen, Qi Zhu, Jian- } \\
\text { peng Xiao, Jianxiang Geng, Zhe Liu, } \\
\text { Jianxiong Hu, Weilin Zeng, Xing } \\
\text { Li, Yuhuang Liao, Xiujuan Tang, } \\
\text { Songjian Xiao, Ying Wang, Yingchao } \\
\text { Song, Xue Zhuang, Liyun Liang, } \\
\text { Guanhao He, Huihong Deng, Tie Song, } \\
\text { Jianfeng He, Wenjun Ma }\end{array}$ \\
\hline $18 / 01 / 20$ & $\begin{array}{l}\text { BetaCoV/Chongqing/IVDC-CQ-00 } \\
1 / 2020\end{array}$ & $\begin{array}{l}\text { National Institute for } \\
\text { Viral Disease Control } \\
\text { and Prevention }\end{array}$ & $\begin{array}{l}\text { National Institute for } \\
\text { Viral Disease Control }\end{array}$ & $\begin{array}{l}\text { Wenjie Tan, Henggin Wang, Xiang } \\
\text { Zhao, Wenling Wang, Peihua Niu, } \\
\text { Roujian Lu, Sheng Ye, Baoying Huang, } \\
\text { Li Zhao, Fei Ye, Wenbo Xu, George F. } \\
\text { Gao, Guizhen Wu }\end{array}$ \\
\hline $18 / 01 / 20$ & BetaCoV/Beijing/IV DC-BJ-005/2020 & $\begin{array}{l}\text { National Institute for } \\
\text { Viral Disease Control } \\
\text { and Prevention }\end{array}$ & $\begin{array}{l}\text { National Institute for } \\
\text { Viral Disease Control }\end{array}$ & $\begin{array}{l}\text { Wenjie Tan, Quanyi Wang, Wenling } \\
\text { Wang, Peihua Niu,Roujian Lu, Yang } \\
\text { Pan,Xiang Zhao,Baoying Huang,Li } \\
\text { Zhao,Fei Ye,Wenbo Xu, George F. } \\
\text { Gao,Guizhen Wu }\end{array}$ \\
\hline $19 / 01 / 20$ & $\mathrm{BetaCoV} / \mathrm{USA} / \mathrm{WA} 1 / 2020$ & $\begin{array}{l}\text { Providence Regional } \\
\text { Medical Center }\end{array}$ & $\begin{array}{l}\text { Division of Viral Dis- } \\
\text { eases, Centers for Dis- } \\
\text { ease Control and Pre- } \\
\text { vention }\end{array}$ & $\begin{array}{l}\text { Queen,K., Tao, Y., Li,Y., Paden,C.R., } \\
\text { Lu,X., Zhang,J., Gerber,S.I., Lind- } \\
\text { strom,S., Tong,S. }\end{array}$ \\
\hline $19 / 01 / 20$ & $\begin{array}{l}\text { BetaCoV/Hangzhou/HZCDC000 } \\
1 / 2020\end{array}$ & $\begin{array}{l}\text { Hangzhou Center for } \\
\text { Disease Control and } \\
\text { Prevention }\end{array}$ & $\begin{array}{l}\text { Hangzhou Center for } \\
\text { Disease Control and } \\
\text { Prevention }\end{array}$ & $\begin{array}{l}\text { Jun Li, Haogiu Wang, Hua Yu, } \\
\text { Lingeng Mao, Xinfen Yu, Zhou Sun, } \\
\text { Qingxin Kong, Xin Qian, Shuchang } \\
\text { Chen, Xuchu Wang }\end{array}$ \\
\hline $19 / 01 / 20$ & $\begin{array}{l}\text { BetaCoV/Shandong/IVDC-SD-00 } \\
1 / 2020\end{array}$ & $\begin{array}{l}\text { National Institute for } \\
\text { Viral Disease Control } \\
\text { and Prevention }\end{array}$ & $\begin{array}{l}\text { National Institute for } \\
\text { Viral Disease Control }\end{array}$ & $\begin{array}{l}\text { Wenjie Tan, Zhaoguo Wang, Xiang } \\
\text { Zhao, Wenling Wang, Peihua Niu, } \\
\text { Roujian Lu, Ti Liu, Baoying Huang, } \\
\text { Li Zhao, Fei Ye, Wenbo Xu, George F. } \\
\text { Gao, Guizhen Wu }\end{array}$ \\
\hline $19 / 01 / 20$ & $\begin{array}{l}\text { BetaCoV/Jiangsu/IVDC-JS-00 } \\
1 / 2020\end{array}$ & $\begin{array}{l}\text { National Institute for } \\
\text { Viral Disease Control } \\
\text { and Prevention }\end{array}$ & $\begin{array}{l}\text { National Institute for } \\
\text { Viral Disease Control }\end{array}$ & $\begin{array}{l}\text { Wenjie Tan, Shenjiao Wang, Wen- } \\
\text { ling Wang, Peihua Niu, Roujian Lu, } \\
\text { Kangchen Zhao, Xiang Zhao, Baoying } \\
\text { Huang, Li Zhao, Fei Ye, Wenbo Xu, } \\
\text { George F. Gao, Guizhen Wu }\end{array}$ \\
\hline $20 / 01 / 20$ & BetaCoV/Zhejiang/Hangzhou- 1/2020 & $\begin{array}{l}\text { Hangzhou Center for } \\
\text { Disease and Control Mi- } \\
\text { crobiology Lab }\end{array}$ & $\begin{array}{l}\text { Hangzhou Center for } \\
\text { Disease and Control Mi- } \\
\text { crobiology Lab }\end{array}$ & $\begin{array}{l}\text { Yu Hua, Wang Haogiu, Li Jun, Yu Xin- } \\
\text { feng }\end{array}$ \\
\hline $21 / 01 / 20$ & BetaCoV/USA/IL $1 / 2020$ & $\begin{array}{l}\text { IL Department of Pub- } \\
\text { lic Health Chicago Lab- } \\
\text { oratory }\end{array}$ & $\begin{array}{l}\text { Pathogen Discovery, } \\
\text { Respiratory Viruses } \\
\text { Branch, Division of } \\
\text { Viral Diseases, Centers } \\
\text { for Dieases Control and }\end{array}$ & X \\
\hline $21 / 01 / 20$ & BetaCoV/Chonggqing/Y C0 1/2020 & $\begin{array}{lr}\text { Yongchuan } & \text { District } \\
\text { Center for } & \text { Disease } \\
\text { Control and Prevention }\end{array}$ & $\begin{array}{l}\text { Chongqing } \begin{array}{r}\text { Municipal } \\
\text { Center for } \\
\text { Control and Prevention }\end{array}\end{array}$ & $\begin{array}{l}\text { Ye Sheng, Tang Yun, Ling Hua, Yu } \\
\text { zhen,Chen Shuang, Tan ZhangPing, Su } \\
\text { Kun, Li Qing, Tang Wenge, Rong } \\
\text { Rong }\end{array}$ \\
\hline $22 / 01 / 20$ & $\mathrm{BetaCoV} / \mathrm{USA} / \mathrm{CA} 2 / 2020$ & $\begin{array}{l}\text { California Department } \\
\text { of Public Health }\end{array}$ & $\begin{array}{l}\text { Pathogen Discovery, } \\
\text { Respiratory Viruses } \\
\text { Branch, Division of } \\
\text { Viral Diseases, Centers } \\
\text { for Dieases Control and }\end{array}$ & \\
\hline $22 / 01 / 20$ & BetaCoV/USA/AZ1/2020 & $\begin{array}{l}\text { Arizona Department of } \\
\text { Health Services }\end{array}$ & $\begin{array}{l}\text { Pathogen Discovery, } \\
\text { Respiratory Viruses } \\
\text { Branch, Division of } \\
\text { Viral Diseases, Centers } \\
\text { for Disease Control and }\end{array}$ & NA \\
\hline $22 / 01 / 20$ & BetaCoV/Guangdong/20SF 174/2020 & $\begin{array}{l}\text { Guangdong Provincial } \\
\text { Center for Piseases } \\
\text { Contorl and Prevention }\end{array}$ & $\begin{array}{l}\text { Guangdong Provincial } \\
\text { Center for Disease } \\
\text { Control and Prevention }\end{array}$ & $\begin{array}{l}\text { Min Kang, Jie Wu, Jing Lu, Tao } \\
\text { Liu, Baisheng Li, Shujiang Mei, Feng } \\
\text { Ruan, Lifeng Lin, Changwen Ke, Hao- } \\
\text { jie Zhong, Yingtao Zhang, Lirong } \\
\text { Zou, Xuguang Chen, Qi Zhu, Jian- } \\
\text { peng Xiao, Jianxiang Geng, Zhe Liu, } \\
\text { Jianxiong Hu, Weilin Zeng, Xing } \\
\text { Li, Yuhuang Liao, Xiujuan Tang, } \\
\text { Songjian Xiao, Ying Wang, Yingchao } \\
\text { Song, Xue Zhuang, Liyun Liang, } \\
\text { Guanhao He, Huihong Deng, Tie Song, } \\
\text { Jianfeng He, Wenjun Ma }\end{array}$ \\
\hline $22 / 01 / 20$ & BetaCoV/Guangzhou/20SF 206/2020 & $\begin{array}{l}\text { Guangdong Provincial } \\
\text { Center for Piseases } \\
\text { Contorl and Prevention }\end{array}$ & $\begin{array}{l}\text { Guangdong Provincial } \\
\text { Center for Diseases } \\
\text { Control and Prevention }\end{array}$ & $\begin{array}{l}\text { Min Kang, Jie Wu, Jing Lu, Tao } \\
\text { Liu, Baisheng Li, Shujiang Mei, Feng } \\
\text { Ruan, Lifeng Lin, Changwen Ke, Hao- } \\
\text { jie Zhong, Yingtao Zhang, Lirong } \\
\text { Zou, Xuguang Chen, Qi Zhu, Jian- } \\
\text { peng Xiao, Jianxiang Geng, Zhe Liu, } \\
\text { Jianxiong Hu, Weilin Zeng, Xing } \\
\text { Li, Yuhuang Liao, Xiujuan Tang, } \\
\text { Songjian Xiao, Ying Wang, Yingchao } \\
\text { Song, Xue Zhuang, Liyun Liang, } \\
\text { Guanhao He, Huihong Deng, Tie Song, } \\
\text { Jianfeng He, Wenjun Ma }\end{array}$ \\
\hline
\end{tabular}


Table 22. Nucleotide sequences used in this study that were obtained from the GISAID EpiFlu Database ${ }^{\top M}$ (contd.).

\begin{tabular}{|c|c|c|c|c|}
\hline Collection date & Sequence id & Originating lab & Submitting lab & Authors \\
\hline $22 / 01 / 20$ & BetaCo V/Foshan/20SF207/2020 & $\begin{array}{l}\text { Guangdong Provincial } \\
\text { Center for Diseases } \\
\text { Control and Prevention }\end{array}$ & $\begin{array}{l}\text { Guangdong Provincial } \\
\text { Center for Diseases } \\
\text { Control and Prevention }\end{array}$ & $\begin{array}{l}\text { Min Kang, Jie Wu, Jing Lu, Tao } \\
\text { Liu, Baisheng Li, Shujiang Me, Feng } \\
\text { Ruan, Lifeng Lin, Changwen Ke, Hao- } \\
\text { jie Zhong, Yingtao Zhang, Lirong } \\
\text { Zou, Xuguang Chen, Qi Zhu, Jian- } \\
\text { peng Xiao, Jianxiang Geng, Zhe Liu, } \\
\text { Jianxiong Hu, Weilin Zeng, Xing } \\
\text { Li, Yuhuang Liao, Xiujuan Tang, } \\
\text { Songjian Xiao, Ying Wang, Yingchao } \\
\text { Song, Xue Zhuang, Liyun Liang, } \\
\text { Guanhao He, Huihong Deng, Tie Song, } \\
\text { Jianfeng He, Wenjun Ma }\end{array}$ \\
\hline $22 / 01 / 20$ & BetaCo V/Foshan/20SF2 10/2020 & $\begin{array}{l}\text { Guangdong Provincial } \\
\text { Center for Diseases } \\
\text { Control and Prevention }\end{array}$ & $\begin{array}{l}\text { Guangdong Provincial } \\
\text { Center for Diseases } \\
\text { Control and Prevention }\end{array}$ & $\begin{array}{l}\text { Min Kang, Jie Wu, Jing Lu, Tao } \\
\text { Liu, Baisheng Li, Shujiang Mei, Feng } \\
\text { Ruan, Lifeng Lin, Changwen Ke, Hao- } \\
\text { jie Zhong, Yingtao Zhang, Lirong } \\
\text { Zou, Xuguang Chen, Qi Zhu, Jian- } \\
\text { peng Xiao, Jianxiang Geng, Zhe Liu, } \\
\text { Jianxiong Hu, Weilin Zeng, Xing } \\
\text { Li, Yuhuang Liao, Xiujuan Tang, } \\
\text { Songjian Xiao, Ying Wang, Yingchao } \\
\text { Song, Xue Zhuang, Liyun Liang, } \\
\text { Guanhao He, Huihong Deng, Tie Song, } \\
\text { Jianfeng He, Wenjun Ma }\end{array}$ \\
\hline $23 / 01 / 20$ & BetaCoV/Foshan/20SF21 1/2020 & $\begin{array}{l}\text { Guangdong Provincial } \\
\text { Center for Diseases } \\
\text { Control and Prevention }\end{array}$ & $\begin{array}{l}\text { Guangdong Provincial } \\
\text { Center for Diseases } \\
\text { Control and Prevention }\end{array}$ & $\begin{array}{l}\text { Min Kang, Jie Wu, Jing Lu, Tao } \\
\text { Liu, Baisheng Li, Shujiang Mei, Feng } \\
\text { Ruan, Lifeng Lin, Changwen Ke, Hao- } \\
\text { jie Zhong, Yingtao Zhang, Lirong } \\
\text { Zou, Xuguang Chen, Qi Zhu, Jian- } \\
\text { peng Xiao, Jianxiang Geng, Zhe Liu, } \\
\text { Jianxiong Hu, Weilin Zeng, Xing } \\
\text { Li, Yuhuang Liao, Xiujuan Tang, } \\
\text { Songjian Xiao, Ying Wang, Yingchao } \\
\text { Song, Xue Zhuang, Liyun Liang, } \\
\text { Guanhao He, Huihong Deng, Tie Song, } \\
\text { Jianfeng He, Wenjun Ma } \\
\text { Ji-Rong Yang, Yu-Chi Lin, Jung-Jung } \\
\text { Mu, Ming-Tsan Liu, Shu- Ying Li }\end{array}$ \\
\hline $23 / 01 / 20$ & BetaCoV/USA/CA1/2020 & $\begin{array}{l}\text { California Department } \\
\text { of Public Health }\end{array}$ & $\begin{array}{l}\text { Pathogen Discovery, } \\
\text { Respiratory Viruses } \\
\text { Branch, Division of } \\
\text { Viral Diseases, Centers } \\
\text { for Dieases Control and }\end{array}$ & NA \\
\hline $23 / 01 / 20$ & BetaCoV/Guangdong/20SF 201/2020 & $\begin{array}{l}\text { Guangdong Provincial } \\
\text { Center for Diseases } \\
\text { Control and Prevention }\end{array}$ & $\begin{array}{l}\text { Guangdong Provincial } \\
\text { Center for Diseases } \\
\text { Control and Prevention }\end{array}$ & $\begin{array}{l}\text { Min Kang, Jie Wu, Jing Lu, Tao } \\
\text { Liu, Baisheng Li, Shujiang Me, Feng } \\
\text { Ruan, Lifeng Lin, Changwen Ke, Hao- } \\
\text { jie Zhong, Yingtao Zhang, Lirong } \\
\text { Zou, Xuguang Chen, Qi Zhu, Jian- } \\
\text { peng Xiao, Jianxiang Geng, Zhe Liu, } \\
\text { Jianxiong Hu, Weilin Zeng, Xing } \\
\text { Li, Yuhuang Liao, Xiujuan Tang, } \\
\text { Songjian Xiao, Ying Wang, Yingchao } \\
\text { Song, Xue Zhuang, Liyun Liang, } \\
\text { Guanhao He, Huihong Deng, Tie Song, } \\
\text { Jianfeng He, Wenjun Ma }\end{array}$ \\
\hline $23 / 01 / 20$ & BetaCo V/France/IDF0372/2020 & $\begin{array}{l}\text { Department of Infec- } \\
\text { tious and } \\
\text { Diseases }\end{array}$ & $\begin{array}{l}\text { National Reference Cen- } \\
\text { ter for Viruses of Respi- } \\
\text { ratory Infections, Insti- } \\
\text { tut Pasteur, Paris }\end{array}$ & $\begin{array}{l}\text { Mélanie Albert, Marion Barbet, Sylvie } \\
\text { Behillil, Méline Bizard, Angela Brise- } \\
\text { barre, Flora Donati, Vincent Enouf, } \\
\text { Maud Vanpeene, Sylvie van der Werf, } \\
\text { Yazdan Yazdanpanah, Xavier Lescure. }\end{array}$ \\
\hline $23 / 01 / 20$ & BetaCoV/France/IDF0373/2020 & $\begin{array}{l}\text { Department } \\
\text { tious and } \text { Infec- } \\
\text { Diseases }\end{array}$ & $\begin{array}{l}\text { National Reference Cen- } \\
\text { ter for Viruses of Respi- } \\
\text { ratory Infections, Insti- } \\
\text { tut Pasteur, Paris }\end{array}$ & $\begin{array}{l}\text { Mélanie Albert, Marion Barbet, Sylvie } \\
\text { Behillil, Méline Bizard, Angela Brise- } \\
\text { barre, Flora Donati, Vincent Enouf, } \\
\text { Maud Vanpeene, Sylvie van der Werf, } \\
\text { Yazdan Yazdanpanah, Xavier Lescure. }\end{array}$ \\
\hline $23 / 01 / 20$ & BetaCoV/Singapore/ 1/2020 & $\begin{array}{l}\text { Singapore General Hos- } \\
\text { pital }\end{array}$ & $\begin{array}{l}\text { National Public Health } \\
\text { Laboratory }\end{array}$ & \\
\hline $23 / 01 / 20$ & BetaCoV/Chonggqing/ZX0 1/2020 & $\begin{array}{l}\text { Zhongxian Center for } \\
\text { Disease Control and } \\
\text { Prevention }\end{array}$ & $\begin{array}{l}\text { Chongqing Municipal } \\
\text { Center for Disease } \\
\text { Control and Prevention }\end{array}$ & $\begin{array}{l}\text { Ye Sheng, Tang Yun, Ling Hua, Zhang } \\
\text { Hong, Yu zhen, Chen Shuang, Tan } \\
\text { ZhangPing, Su Kun, Li Qin, Tang } \\
\text { Wenge, Rong Rong }\end{array}$ \\
\hline $24 / 01 / 20$ & BetaCoV/Australia/NS WO $1 / 2020$ & $\begin{array}{lrr}\text { Centre } & \text { for } & \text { Infectious } \\
\text { Diseases } & \text { and Micro- } \\
\text { biology } & \text { Laboratory } \\
\text { Services } & \end{array}$ & $\begin{array}{l}\text { NSW Health Pathology } \\
\text { - Institute of Clinical } \\
\text { Pathology and Medical } \\
\text { Research }\end{array}$ & NA \\
\hline $25 / 01 / 20$ & BetaCoV/Australia/VICO1/2020 & Monash Medical Centre & $\begin{array}{l}\text { Collaboration between } \\
\text { the University of Mel- } \\
\text { bourne at The Peter Do- } \\
\text { herty Institute for Infec- } \\
\text { tion and Immunity, and }\end{array}$ & $\begin{array}{l}\text { Caly,L., Seemann,T., } \\
\text { Druce,J. and Taiaroa,G }\end{array}$ \\
\hline $25 / 01 / 20$ & BetaCoV/Japan/AI/I-004/2020 & $\begin{array}{l}\text { Department of Virology } \\
\text { III }\end{array}$ & 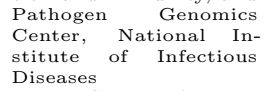 & $\begin{array}{l}\text { Tsuyoshi Sekizuka, Shutoku Mat- } \\
\text { suyama, Naganori Nao, Kazuya } \\
\text { Shirato, Shinji Watanabe, Makoto } \\
\text { Takeda, Makoto Kuroda }\end{array}$ \\
\hline $25 / 01 / 20$ & BetaCoV/Korea/KCDC03/2020 & $\begin{array}{l}\text { Korea Centers for Dis- } \\
\text { ease Control }\end{array}$ & $\begin{array}{l}\text { Korea Centers for Dis- } \\
\text { ease Control }\end{array}$ & $\begin{array}{l}\text { Jeong-Min Kim, Yoon-Seok Chung, } \\
\text { Namjoo Lee, Mi-Seon Kim, SangHee } \\
\text { Woo, Hye-Joon Jo, Sehee Park, Heui } \\
\text { Man Kim, Myung Guk Han }\end{array}$ \\
\hline $25 / 01 / 20$ & BetaCoV/USA/WA1-A12/2020 & $\begin{array}{l}\text { WA State Department } \\
\text { of Health }\end{array}$ & $\begin{array}{l}\text { Pathogen Discovery, } \\
\text { Respiratory Viruses } \\
\text { Branch, Division of } \\
\text { Viral Diseases, Centers } \\
\text { for Dieases Control and }\end{array}$ & NA \\
\hline $25 / 01 / 20$ & $\mathrm{BetaCoV} / \mathrm{USA} / \mathrm{W}$ A 1-F6/2020 & $\begin{array}{l}\text { WA State Department } \\
\text { of Health }\end{array}$ & $\begin{array}{l}\text { Pathogen Discovery, } \\
\text { Respiratory Viruses } \\
\text { Branch, Division of } \\
\text { Viral Diseases, Centers } \\
\text { for Dieases Control and }\end{array}$ & NA \\
\hline $25 / 01 / 20$ & BetaCoV/Singapore/2/2020 & $\begin{array}{l}\text { Singapore General Hos- } \\
\text { pital }\end{array}$ & $\begin{array}{l}\text { Programme in Emerg- } \\
\text { ing Infectious Diseases, } \\
\text { Duke-NUS Medical } \\
\text { School }\end{array}$ & $\begin{array}{l}\text { Danielle E Anderson, Martin Lin- } \\
\text { ster, Yan Zhuang, Jayanthi Jayaku- } \\
\text { mar, Kian Sing Chan, Lynette LE Oon, } \\
\text { Jenny GH Low, Yvonne CF Su, Linfa } \\
\text { Wang, Gavin JD Smith }\end{array}$ \\
\hline
\end{tabular}


Table 23. Nucleotide sequences used in this study that were obtained from the GISAID EpiFlu Database ${ }^{\mathrm{TM}}$ (contd.).

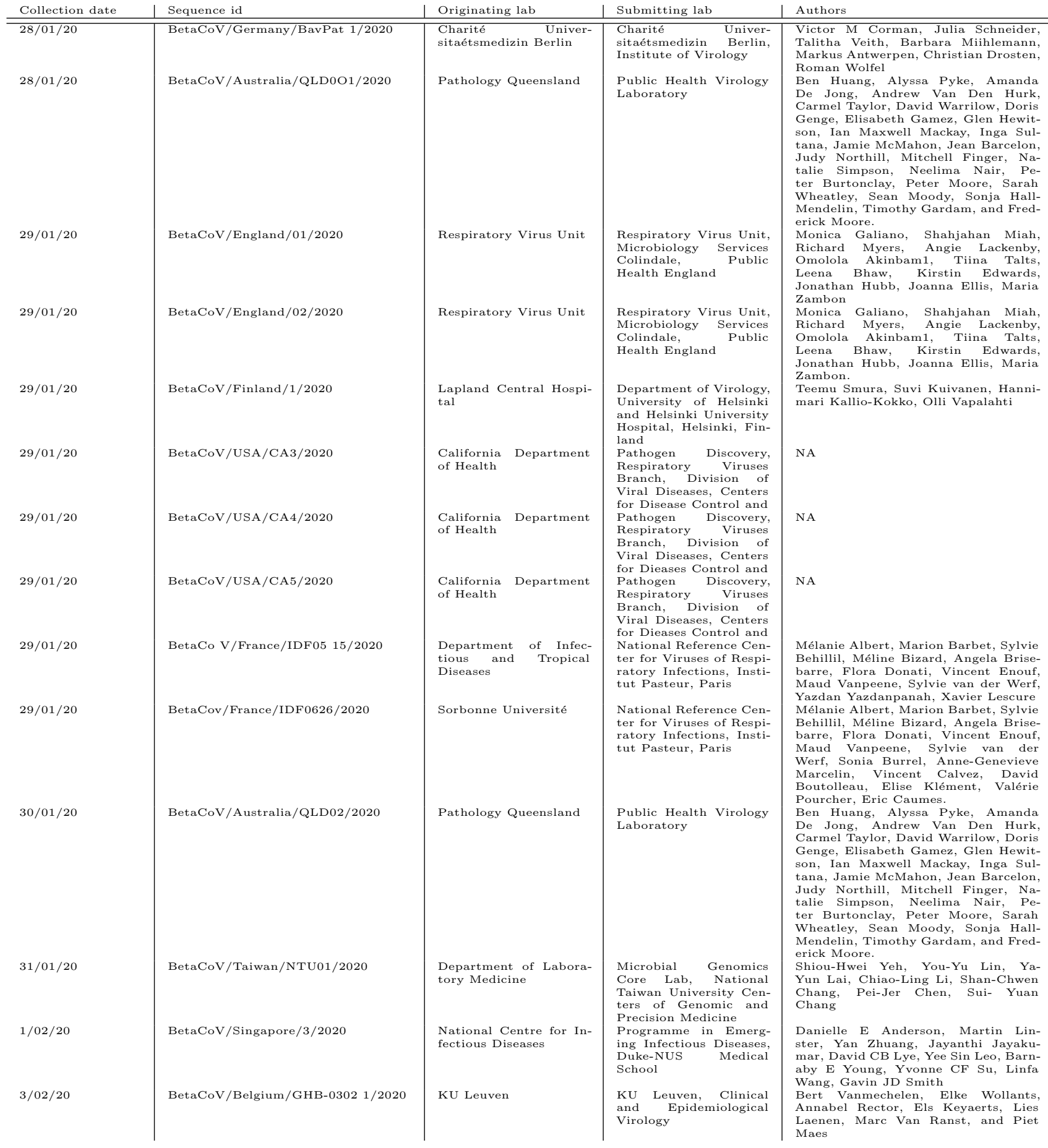

